\title{
A Review on Bamboo Resource in the African Region: A Call for Special Focus and Action
}

\author{
Tinsae Bahru (i) ${ }^{1}$ and Yulong Ding ${ }^{2}$ \\ ${ }^{1}$ Central Ethiopia Environment and Forest Research Center (CEE-FRC), P.O. Box 33042, Addis Ababa, Ethiopia \\ ${ }^{2}$ Bamboo Research Institute, Nanjing Forestry University, Nanjing 210037, China \\ Correspondence should be addressed to Tinsae Bahru; batinsae@gmail.com
}

Received 3 September 2020; Revised 16 February 2021; Accepted 25 February 2021; Published 8 March 2021

Academic Editor: Ahmad A. Omar

Copyright (c) 2021 Tinsae Bahru and Yulong Ding. This is an open access article distributed under the Creative Commons Attribution License, which permits unrestricted use, distribution, and reproduction in any medium, provided the original work is properly cited.

\begin{abstract}
The African region has untapped bamboo resource potential with immense socioeconomic, cultural, and ecological significances. Despite the long history of bamboo in the region, its contribution is at the infant stage. Therefore, the present study aimed at reviewing the existing literature supported by research experience on bamboo resource in the region. The review process mainly focused on four main specific objectives. These include (1) review extensively African countries that owned the resource and identify the species in each country, (2) identify and document species, generic, and taxonomic tribes of each bamboo species, (3) assess and report bamboo area coverage from available nations, and (4) highlight the existing experiences of special opportunities, challenges, and successful achievements on bamboo resource in representative African countries. The review process found out that a total of 4.56 million ha total bamboo area and 115 bamboo species are reported from 48 African countries. Hence, the African region shares $12.3 \%$ of the global bamboo resource and contributed $7.3 \%$ of the total bamboo species. Of this, $89.6 \%$ of the region is endowed with indigenous bamboo species. Among indigenous species, O. abyssinica is the most widely distributed in 38 African countries. Madagascar ranked first with 37 indigenous species, while Ethiopia led by 25 introduced bamboo species. Nowadays, Ethiopia has 1.44 million ha total indigenous bamboo area coverage, which accounted for $31.6 \%$ of the African region and $3.89 \%$ of the world total. Therefore, more detail and comprehensive research on species taxonomy, resource base inventory, silvicultural applications, and socioeconomic study is recommended.
\end{abstract}

\section{Global Bamboo Resource Overview}

Bamboo belongs to the subfamily Bambusoideae and family Gramineae or Poaceae [1-3]. Various sources speculated the origin of bamboo in the evolutionary line of plant kingdom. However, Clark estimated that bamboo origin was traced back probably some $30-40$ million years ago [4]. It is one of the most important forest resources with immense socioeconomic, cultural, and ecological significances since ancient times. For instance, indigenous bamboo resource in Ethiopia has been used for different traditional uses including house construction, fencing, production of handicrafts and other household utensils, animal feed, edible shoots for human consumption, and many other uses. In the human history, bamboo cultivation and utilization in ancient China traced back to about 6000 years [5]. As early as 3000 years before, edible bamboo shoot has been used as a popular and delicious dish in China [6]. Nowadays, there are 1575 bamboo species [1] belonging to about 90 genera across the world $[4,7]$. The bamboo resource further covers a total area of about 37 million ha worldwide or around $1 \%$ of the global forest resource [7]. Its annual production also accounted for more than 20 million tons [8] and contributed $\$ 60$ billion to the global economy [9]. In relation to this, bamboo resource is widely distributed around the world in diverse climatic and ecological settings. Its range covers from tropics, subtropics, and temperate to frigid zones [4, 10-12] approximately in $50^{\circ} \mathrm{N}-47^{\circ} \mathrm{S}$ [4]. The altitudinal ranges also vary from the sea level up to a higher elevation, i.e., $4500 \mathrm{~m}$ above the sea level [1]. 
As a whole, the global distribution of bamboo resource can be classified into four major geographic regions [10-12] (Figure 1). These major bamboo regions comprise the AsiaPacific region with more than 900 species $[10,11]$, American region with over 500 species [4], and the African region with 43 species [13-15]. The African region comprises the mainland Africa and the associated islands surrounding the continent including Comoros, Madagascar, Mauritius, Réunion, São Tomé and Príncipe, and Seychelles. These regions are specifically located at $51^{\circ} \mathrm{N}-42^{\circ} \mathrm{S}$ [12], $40^{\circ} \mathrm{N}-47^{\circ} \mathrm{S}$ $[10-12]$, and $16^{\circ} \mathrm{N}-22^{\circ} \mathrm{S}[10,11,16]$ in their respective orders. By contrast, European, North American, and Australian regions are emerged due to the introduction of many bamboo species from Asia, Africa, and South America mostly for gardening, ornamentals, and other uses [10-12].

In this insight, approximately $80 \%$ of the bamboo resource is found in the Asia-Pacific region [10, 11]. Of this, more than $59 \%$ of the Asia-Pacific region $[3,10,11]$ and $33.9 \%$ of the world bamboo species are found in China $[1,3]$. Currently, 534 bamboo species that belong to 34 genera are found in China [3] with the total area coverage of 7 million ha $[11,16]$. In contrast, the African region has very little bamboo resource in terms of species diversity and area coverage almost entirely limited to tropical zones [4]. It comprises $7 \%$ of the world bamboo resource with total area coverage of over 2.8 million ha within six nations [7]. Therefore, it needs urgent call for special focus and action for the sustainable development and promotion of bamboo resource in the African region. This comprises (1) review extensively African countries that owned the resource and identify the species in each country by their scientific names, (2) identify and document bamboo species including their description and generic and taxonomic tribes of each bamboo species, (3) assess and report bamboo area coverage from African nations that have available information, and (4) highlight special opportunities, challenges, and successful achievements on bamboo resource in representative African countries.

\section{Materials and Methods}

A comprehensive and detail literature review was carried out from 108 published and accessed bibliographical sources. These included 54 scientific journals, 15 books, 19 official documents from various nations and/or organizations and working studies, 11 workshop proceedings, manuals, and newspapers, 4 online accessed resources, and 5 academic theses. The review process encompassed both African countries and islands surrounding the mainland Africa. Consequently, the total area covered wider and diverse geographical locations and settings, altitudinal ranges, climatic conditions, and socioeconomic and cultural diversities and lifestyles. At the same time, the perception, experience, and knowledge of local people and nations focus towards bamboo resource are considerably varied. With this in mind, available data in each country were reviewed in detail, bamboo species were identified, and a species list was documented. Thereafter, the scientific names and their synonymous if any were listed down and particular references are cited. After that, the generic names are identified and grouped under taxonomic tribes following different references. In the same way, regarding to the total bamboo area coverage in the region, data from available countries were extensively reviewed, and then, the countries list, bamboo area, and bamboo area to forest area coverage as well as percentage share are presented. The existing practical experiences on widely distributed, commonly used, and potentially high species are selected as representative species and extensively reviewed. Last, special opportunities, major challenges, and successful achievements are assessed from typical countries so as to strengthen the resource development and promotion in the region.

\section{Origin and Distribution of Bamboo Resource in the African Region}

Our extensive literature review showed that a total of 115 bamboo species are widely distributed among 48 countries in the African region (Table 1). This accounted for $7.3 \%$ of the global bamboo species and covered $82.8 \%$ of the African region. This covered vast areas which extend from western coast at Senegal to the eastern part at Mauritius, while it stretched from Morocco in the north to South Africa in the southern part. Out of the indigenous bamboo species, Oxytenanthera abyssinica is widely distributed among 38 countries, while Olyra latifolia is found within 30 countries. These are followed by Oldeania alpina and Oreobambos buchwaldii, which are further recorded among 13 and 10 African countries, respectively. In the same way, 5 countries have Guaduella oblonga, while Bambusa vulgaris, Guaduella densiflora, and Hickelia africana are recorded among the 3 African countries, each. Regarding to introduced bamboo species, Bambusa vulgaris is widely distributed among 20 African countries, followed by Dendrocalamus giganteus within 10 countries. Also, $D$. asper and D. strictus are equally found in 6 countries, each (Table 1).

In relation to bamboo genera, the genus Bambusa contained 25 bamboo species, which accounted for $21.7 \%$ of the total recorded species in the region (Table 2). This is followed by the genus Nastus and Dendrocalamus with 12 and 11 bamboo species, respectively. Similarly, 6 bamboo species are classified under the genus Guaduella, whereas 5 species are recorded under the genus Yushania. The genera Cephalostachyum and Hickelia followed with 4 species, each.

In the same way, a total of 35 bamboo genera are recorded in the African region, which are classified under five taxonomic tribes (Table 3). Of these, the tribe Bambuseae comprised a total of 19 (54.3\%) bamboo genera, followed by Arundinarieae with 11 taxonomic genera. On the other hand, three tribes, namely, Guaduelleae, Olyreae, and Puelieae, contained one bamboo genera, each. In contrast, there is no concrete information available to group the remaining two bamboo genera to a given tribe and hence requires a further taxonomic study. In line with this, tribe Bambuseae is distributed among the 45 African nations and Olyreae is distributed among 30 nations (Table 3 ). 


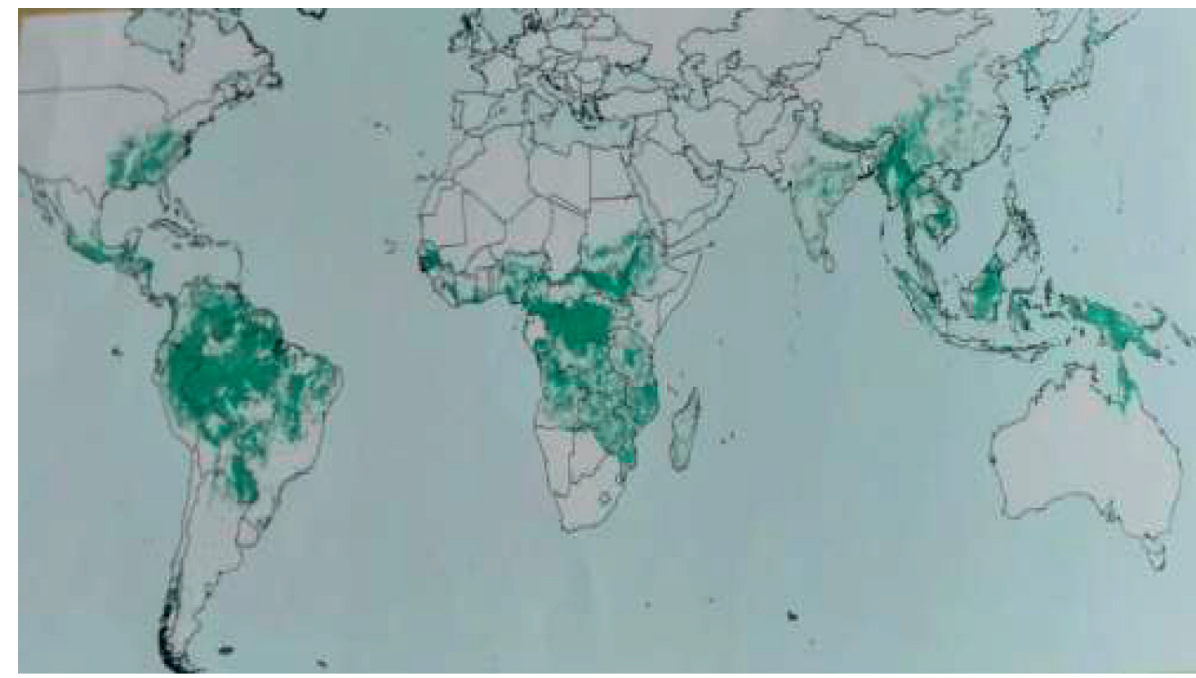

FIGURE 1: Global bamboo resource distribution among the four major geographic regions (source: [12]).

Furthermore, comprehensive literature review confirmed that the distribution of bamboo resource in the African region can be classified broadly into two parts. These are the mainland Africa and the associated six islands surrounding the continent (Comoros, Madagascar, Mauritius, Réunion, São Tomé and Príncipe, and Seychelles). The review process reported that mainland Africa has 83 bamboo species belonging to 30 genera (Table 4). Out of these species, 20 bamboo species are indigenous (native) to the region. The remaining 63 species are mainly introduced (exotic) from other regions (Asia-Pacific, America, or Africa itself). On the other hand, the six islands comprise 50 species. Among these, 40 bamboo species are indigenous, while 10 of them are introduced from elsewhere. Therefore, majority of the bamboo species $(72.2 \%)$ are introduced to the mainland Africa at various times. By contrast, more diverse indigenous bamboo species (34.8\%) are found within the six associated islands. This clearly shows that the mainland Africa has less rich and diverse indigenous compared to introduced bamboo species.

In the same way, a total of up to 45 bamboo species are reported from each country (Table 5). In terms of species origin, bamboo species are classified as indigenous to Africa or introduced from elsewhere. Among these, 22 countries have only indigenous (native) bamboo species, 5 countries have only introduced (exotic) species, while 21 countries owned both species. This reflects that $89.6 \%$ of the region is endowed with indigenous bamboo species. From indigenous species, Madagascar ranked first with a total of 37 bamboo species (refer Table 1), followed by Cameroon with 10 species (Table 1). This clearly shows that the species diversity and distribution at Madagascar is much richer than the mainland Africa $[4,12]$. Ghana and Tanzania also comprise 8 and 6 species, respectively (Table 1). On the other hand, Ethiopia ranked first with a total of 25 introduced bamboo species, followed by Togo with 20 species. Ghana and Kenya each contains with a total of 16 introduced species, whereas
Nigeria and Sudan follow with 12 and 10 bamboo species, respectively.

\section{Status and Potential of Bamboo Resource in the African Region}

The status and potential of bamboo resource in the African region is reviewed from different sources. According to the reports, the data are only available from 12 African countries. In this insight, $12.3 \%$ of the global bamboo resource is contributed by the African region. This indicated that bamboo development in the region is slightly improved as compared to $7 \%$ of total bamboo resource reported by FAO [7]. Ethiopia shares $31.55 \%$ of the total bamboo resource in the African region, followed by Senegal (14.49\%) and Ghana $(8.77 \%)$ (Table 6). Similarly, the bamboo to forest area coverage accounted for $11.51 \%, 7.99 \%$, and $4.28 \%$ in their respective orders. However, the figure reported from Nigeria $(34.88 \%)$ is an overestimated data and hence not yet verified [7]. By contrast, available data from Cameroon [23] and Zimbabwe (FAO (2001) cited in FAO [7] are also incomplete and do not represent the entire countries' resource. But, the intention to include these data is to show the resource potential and thereby to give more focus to the region. In the same way, the bamboo resource reported from other countries in the region (Tables 1 and 2) is not well known and estimated. Therefore, we concluded that due attention should be given to the status and potential of bamboo resource in the African region.

\section{Overview on Indigenous Bamboo Species in Ethiopia}

Out of the total recorded indigenous bamboo species in the African region (60 species), two indigenous bamboo species (O. abyssinica and O. alpina) are widely distributed and commonly used in the region, and their origin also traced 
TABLe 1: Origin and distribution of bamboo resource in the African region.

\begin{tabular}{|c|c|c|c|c|}
\hline \multirow{2}{*}{ No. } & \multirow{2}{*}{ List of countries } & \multicolumn{2}{|c|}{ Origin and distribution of bamboo resource in the African region } & \multirow{2}{*}{ Reference } \\
\hline & & Indigenous (native) species & Introduced (exotic) species & \\
\hline 1 & Algeria & & Pseudosasa japonica & INBAR [17] \\
\hline 2 & Angola & $\begin{array}{c}\text { Guaduella densiflora, Guaduella } \\
\text { dichroa, Olyra latifolia, Oreobambos } \\
\text { buchwaldii, and Oxytenanthera } \\
\text { abyssinica. }\end{array}$ & & $\begin{array}{c}\text { Ohrnberger [1], Bystriakova } \\
\text { et al. [18], INBAR [17], Clayton } \\
\text { et al. [19] }\end{array}$ \\
\hline 3 & Benin & O. latifolia and O. abyssinica. & $\begin{array}{c}\text { Bambusa vulgaris, Dendrocalamus } \\
\text { asper, and D. giganteus. }\end{array}$ & $\begin{array}{l}\text { Ohrnberger [1], Bystriakova } \\
\text { et al. [18], zhou [16], Inada and } \\
\text { Hall [20], INBAR [17], Clayton } \\
\text { et al. [19] }\end{array}$ \\
\hline 4 & Burkina Faso & O. latifolia and O. abyssinica. & B. vulgaris & $\begin{array}{c}\text { Inada and Hall [20], INBAR } \\
\text { [17], Clayton et al. [19] }\end{array}$ \\
\hline 5 & Burundi & $\begin{array}{l}\text { Oldeania alpina, O. latifolia, } \\
\text { O. buchwaldii, and O. abyssinica. }\end{array}$ & & $\begin{array}{l}\text { Phillips [2], Bystriakova et al. } \\
\text { [18], Inada and Hall [20], } \\
\text { INBAR [17], Clayton et al. [19] }\end{array}$ \\
\hline 6 & Cameroon & $\begin{array}{l}\text { G. densiflora, Guaduella humilis, } \\
\text { Guaduella macrostachys, Guaduella } \\
\text { marantifolia, Guaduella oblonga, } \\
\text { O. alpina, O. latifolia, O. buchwaldii, } \\
\text { O. abyssinica, and Puelia atractocarpa. }\end{array}$ & $\begin{array}{l}\text { B. vulgaris (B. vulgaris var. vittata } \\
\text { (yellow variety)), Ochlandra } \\
\text { travancorica, and Phyllostachys aurea. }\end{array}$ & $\begin{array}{l}\text { Phillips [2], Grimshaw [21], } \\
\text { Ohrnberger [1], Bystriakova } \\
\text { et al. [18], INBAR [22], Ingram } \\
\text { et al. [23], INBAR [17], Clayton } \\
\text { et al. [19] }\end{array}$ \\
\hline 7 & $\begin{array}{l}\text { Central African } \\
\text { Republic }\end{array}$ & $O$. latifolia and $O$. abyssinica. & & $\begin{array}{c}\text { Ohrnberger [1], Bystriakova } \\
\text { et al. [18], INBAR [17], Clayton } \\
\text { et al. [19] }\end{array}$ \\
\hline 8 & Chad & O. abyssinica & & $\begin{array}{l}\text { KFRI [24], INBAR [17], } \\
\text { Clayton et al. [19] }\end{array}$ \\
\hline 9 & Comoros & O. latifolia and Sirochloa parvifolia. & & Ohrnberger [1], INBAR [17] \\
\hline 10 & Cote d'Ivoire & $\begin{array}{l}\text { G. oblonga, O. latifolia, and } \\
\text { O. abyssinica. }\end{array}$ & B. vulgaris & $\begin{array}{c}\text { Ohrnberger [1], Bystriakova } \\
\text { et al. [18], INBAR [17], Clayton } \\
\text { et al. [19] }\end{array}$ \\
\hline 11 & $\begin{array}{l}\text { Democratic } \\
\text { Republic of } \\
\text { Congo }\end{array}$ & $\begin{array}{l}\text { O. alpina, O. latifolia, O. buchwaldii, } \\
\text { and O. abyssinica. }\end{array}$ & B. vulgaris and D. asper. & $\begin{array}{c}\text { Phillips [2], Ohrnberger [1], } \\
\text { Bystriakova et al. [18], Inada } \\
\text { and Hall [20], INBAR [17], } \\
\text { Clayton et al. [19] }\end{array}$ \\
\hline 12 & Egypt & & B. multiplex (B. nana) and B. vulgaris. & Moustafa et al. [25] \\
\hline 13 & $\begin{array}{l}\text { Equatorial } \\
\text { Guinea }\end{array}$ & O. latifolia and O. abyssinica. & & INBAR [17] \\
\hline
\end{tabular}

$14 \quad$ Eritrea

O. abysinica

Gigantochloa felix, O. alpina, O. latifolia, and O. abyssinica.

Gabon
G. densiflora, G. marantifolia, and G. oblonga.

O. latifolia and O. abyssinica
B. balcooa, B. bambos, B. emeiensis, B. multiplex, B. multiplex

'Albovariegata,' B. oldhamii, B. pachinensis, B. tulda, B. vulgaris (B. vulgaris var. green (green variety), $B$. vulgaris var. striata, and $B$. vulgaris var. vittata), D. asper, D. barbatus,

D. brandisii, D. giganteus, D. hamiltonii, Fu et al. [11], Jiang and Liu [12],

D. latiflorus, D. membranaceus,

D. peculiaris, Gigantochloa apus,

Gigantochloa atter, Gigantochloa

sumatra, Guadua amplexifolia, Guadua

angustifolia, Phyllostachys edulis,

Schizostachyum jaculans, and Thyrsostachys siamensis.
Phillips [2], Ohrnberger [1], Bystriakova et al. [18], Clayton et al. [19]

Chen et al. [10], Huojin [15],

INBAR [17], Clayton et al. [19]

\section{Ohrnberger [1]}

Ohrnberger [1], Bystriakova et al. [18], INBAR [17], Clayton et al. [19] 
TABle 1: Continued.

\begin{tabular}{|c|c|c|c|c|}
\hline \multirow{2}{*}{ No. } & \multirow{2}{*}{ List of countries } & \multicolumn{2}{|c|}{ Origin and distribution of bamboo resource in the African region } & \multirow{2}{*}{ Reference } \\
\hline & & Indigenous (native) species & Introduced (exotic) species & \\
\hline 18 & Ghana & $\begin{array}{l}\text { B. bambos, B. multiplex, } \\
\text { B. pervariabilis, B. vulgaris }(B . \text { vulgaris } \\
\text { var. green and B. vulgaris var. vittata), } \\
\text { D. strictus, G. macrostachys, O. latifolia, } \\
\text { and O. abysinica. }\end{array}$ & $\begin{array}{c}\text { B. burmanica, B. heterostachya, } \\
\text { B. oldhamii, B. textilis, B. ventricosa, } \\
\text { D. asper, D. barbatus, D. brandisii, } \\
\text { D. giganteus, D. latiflorus, } \\
\text { D. membranaceus, Gigantochloa } \\
\text { albociliata, G. angustifolia, Guadua } \\
\text { chacoensis, P. edulis, and T. siamensis. }\end{array}$ & $\begin{array}{c}\text { Ohrnberger [1], Bystriakova } \\
\text { et al. [18], Inada and Hall [20], } \\
\text { Appiah-Kubi et al. [26], INBAR } \\
\text { [27], Clayton et al. [19] }\end{array}$ \\
\hline 19 & Guinea & $\begin{array}{l}\text { G. oblonga, O. latifolia, and } \\
\text { O. abyssinica. }\end{array}$ & B. vulgaris & $\begin{array}{c}\text { Ohrnberger [1], Bystriakova } \\
\text { et al. [18], INBAR [17], Clayton } \\
\text { et al. [19] }\end{array}$ \\
\hline 20 & Guinea-Bissau & O. latifolia, and O. abyssinica & & \\
\hline 21 & Kenya & $\begin{array}{l}\text { Hickelia africana, O. alpina, O. latifolia, } \\
\text { O. buchwaldii, and Pseudosasa amabilis }\end{array}$ & $\begin{array}{c}\text { B. bambos, B. lako, B. nutans, B. tulda, } \\
\text { B. vulgaris (B. vulgaris var. vittata), } \\
\text { D. asper, D. brandisii, D. giganteus, } \\
\text { D. hamiltonii, D. membranaceus, } \\
\text { D. strictus, O. abyssinica, P. edulis, } \\
\text { P. nigra var. henonis, Schizostachyum } \\
\text { pergracile, Shibataea kumasaca, and } \\
\text { T. siamensis. }\end{array}$ & $\begin{array}{l}\text { Kigomo and Kamiri [28], } \\
\text { Grimshaw [21], zhou [16], Fu } \\
\text { et al. [11], Jiang and Liu [12], } \\
\text { Kigomo [29], Inada and Hall } \\
\text { [20], KFRI [24], Chen et al. [10], } \\
\text { INBAR [17], Clayton et al. [19] }\end{array}$ \\
\hline 22 & Lesotho & $\begin{array}{c}\text { O. abyssinica, Bergbambos tessellata, } \\
\text { and Thamnocalamus sp. }\end{array}$ & & $\begin{array}{l}\text { Ohrnberger [1], Bystriakova } \\
\text { et al. [18], INBAR [17] }\end{array}$ \\
\hline 23 & Liberia & $\begin{array}{l}\text { G. oblonga, O. latifolia, and } \\
\text { O. abyssinica. }\end{array}$ & & $\begin{array}{c}\text { Ohrnberger [1], Inada and Hall } \\
\text { [20], INBAR [17] }\end{array}$ \\
\hline 24 & Libya & & B. vulgaris & \\
\hline 25 & Madagascar & $\begin{array}{c}\text { Cathariostachys capitata, } \\
\text { Cathariostachys madagascariensis, } \\
\text { Cephalostachyum chapelieri, } \\
\text { Cephalostachyum perrieri, } \\
\text { Cephalostachyum sp., Cephalostachyum } \\
\text { viguieri, Decaryochloa diadelpha, } \\
\text { Hickelia alaotrensis, Hickelia } \\
\text { madagascariensis, Hickelia perrieri, } \\
\text { Hitchcockella baronii, Nastus } \\
\text { ambrensis, N. aristatus, N. decaryanus, } \\
\text { N. elongatus, N. emirnensis, } \\
\text { N. humbertianus, N. lokohoensis, } \\
\text { N. madagascariensis, } \\
\text { N. manongarivensis, N. perrieri, } \\
\text { N. tsaratananensis, Ochlandra capitata, } \\
\text { O. latifolia, Perrierbambus } \\
\text { madagascariensis, Perrierbambus } \\
\text { tsarasaotrensis, Schizostachyum } \\
\text { perrieri, Sirochloa parvifolia } \\
\text { (Schizostachyum bosseri), } \\
\text { Thamnocalamus ibityensis, } \\
\text { Thamnocalamus sp., Yushania } \\
\text { humbertii, Y. madagascariensis, } \\
\text { Y. perrieri, Yushania sp., Valiha diffusa, } \\
\text { V. perrieri, and Valiha sp. }\end{array}$ & $\begin{array}{c}\text { B. multiplex, B. spinosa, B. vulgaris } \\
\text { (B. madagascariensis, B. vulgaris var. } \\
\text { green, and B. vulgaris var. vittata), } \\
\text { D. asper, D. giganteus, D. strictus, } \\
\text { Gigantochloa aff. pseudoarundinacea, } \\
\text { and P. aurea. }\end{array}$ & $\begin{array}{c}\text { Ohrnberger [1], Bystriakova } \\
\text { et al. [18], Inada and Hall [20], } \\
\text { King et al. [30], INBAR [17], } \\
\text { Clayton et al. [19] }\end{array}$ \\
\hline 26 & Malawi & $\begin{array}{l}\text { O. alpina, O. latifolia, O. buchwaldii, } \\
\text { and O. abyssinica. }\end{array}$ & & $\begin{array}{c}\text { Phillips [2], Grimshaw [21], } \\
\text { Ohrnberger [1], Bystriakova } \\
\text { et al. [18], Sosola-Banda and } \\
\text { Johnsen [31] }\end{array}$ \\
\hline 27 & Mali & O. abyssinica & & $\begin{array}{c}\text { Inada and Hall [20], INBAR } \\
\text { [17] }\end{array}$ \\
\hline 28 & Mauritius & Probably $B$. tessellata & B. multiplex and D. giganteus. & Ohrnberger [1], INBAR [17] \\
\hline 29 & Morocco & & P. japonica & INBAR [17] \\
\hline
\end{tabular}


TABle 1: Continued.

\begin{tabular}{|c|c|c|c|c|}
\hline \multirow{2}{*}{ No. } & \multirow{2}{*}{ List of countries } & \multicolumn{2}{|c|}{ Origin and distribution of bamboo resource in the African region } & \multirow{2}{*}{ Reference } \\
\hline & & Indigenous (native) species & Introduced (exotic) species & \\
\hline 30 & Mozambique & $\begin{array}{l}\text { O. latifolia, O. buchwaldii, and } \\
\text { O. abyssinica. }\end{array}$ & $\begin{array}{l}\text { B. bambos, B. vulgaris (B. striata), } \\
\text { D. hamiltonii, and D. strictus. }\end{array}$ & $\begin{array}{c}\text { Ohrnberger [1], Bystriakova } \\
\text { et al. [18], INBAR [17], Clayton } \\
\text { et al. [19] }\end{array}$ \\
\hline 31 & Niger & O. abyssinica & & INBAR [17] \\
\hline 32 & Nigeria & $\begin{array}{l}\text { G. densiflora, G. humilis, O. latifolia, } \\
\text { and O. abyssinica. }\end{array}$ & $\begin{array}{l}\text { B. vulgaris, Brachystachyum stellatus, } \\
\text { Dayeteng spp., D. giganteus, D. sinicus, } \\
\text { Fargesia robusta, Gelidocalamus } \\
\text { stellatus, Nuomizhu xiaoyeteng, P. edulis } \\
\text { (P. heterocycla var. pubescens), } \\
\text { Pleioblastus fortunei, Shibataea } \\
\text { chinensis, and Y. baishazuensis. }\end{array}$ & $\begin{array}{c}\text { Ohrnberger [1], Bystriakova } \\
\text { et al. [18], INBAR [17], Clayton } \\
\text { et al. [19] }\end{array}$ \\
\hline 33 & $\begin{array}{l}\text { Republic of } \\
\text { Congo }\end{array}$ & $\begin{array}{c}\text { G. marantifolia, O. alpina, O. latifolia, } \\
\text { and O. abyssinica. }\end{array}$ & & $\begin{array}{c}\text { Ohrnberger [1], Bystriakova } \\
\text { et al. [18], INBAR [17], Clayton } \\
\text { et al. [19] }\end{array}$ \\
\hline 34 & Réunion & N. borbonicus & D. giganteus & $\begin{array}{c}\text { Inada and Hall [20], INBAR } \\
\text { [17] }\end{array}$ \\
\hline 35 & Rwanda & $O$. alpina and $O$. abyssinica. & B. vulgaris & $\begin{array}{c}\text { Phillips [2], Inada and Hall } \\
\text { [20], INBAR [17], Clayton et al. } \\
\text { [19] }\end{array}$ \\
\hline 36 & $\begin{array}{l}\text { São Tomé and } \\
\text { Príncipe }\end{array}$ & O. latifolia and $O$. abyssinica. & B. balcooa and B. vulgaris. & INBAR [17], Haroun et al. [32] \\
\hline 37 & Senegal & O. latifolia and $O$. abyssinica & & $\begin{array}{c}\text { Phillips [2], Ohrnberger [1], } \\
\text { Bystriakova et al. [18], Inada } \\
\text { and Hall [20], INBAR [17], } \\
\text { Clayton et al. [19] }\end{array}$ \\
\hline 38 & Seychelles & & $\begin{array}{c}\text { B. multiplex, B. vulgaris, D. giganteus, } \\
\text { D. strictus, and P. nigra. }\end{array}$ & Zhou [16], INBAR [17] \\
\hline 39 & Sierra Leone & $\begin{array}{l}\text { G. oblonga, O. latifolia, and } \\
\text { O. abyssinica. }\end{array}$ & B. vulgaris & $\begin{array}{c}\text { Ohrnberger [1], Bystriakova } \\
\text { et al. [18], INBAR [17], Clayton } \\
\text { et al. [19] }\end{array}$ \\
\hline 40 & South Africa & O. abysinica and B. tessellata. & B. balcooa & $\begin{array}{l}\text { Ohrnberger [1], Bystriakova } \\
\text { et al. [18], Inada and Hall [20] }\end{array}$ \\
\hline 41 & South Sudan & O. alpina and $O$. abyssinica. & & $\begin{array}{l}\text { Ohrnberger [1], Bystriakova } \\
\text { et al. [18], Clayton et al. [19] }\end{array}$ \\
\hline 42 & Sudan & O. alpina, O. latifolia, and O. abyssinica. & $\begin{array}{l}\text { B. polymorpha, B. teres, B. tulda, } \\
\text { B. vulgaris, D. giganteus, D. hamiltonii, } \\
\text { D. longispathus, D. strictus, S. pergracile, } \\
\text { and Melocanna baccifera. }\end{array}$ & $\begin{array}{c}\text { Phillips [2], Ohrnberger [1]; } \\
\text { Bystriakova et al. [18]; zhou } \\
\text { [16]; INBAR [17]; Clayton et al. } \\
\text { [19] }\end{array}$ \\
\hline 43 & Swaziland & O. abyssinica & & INBAR [17] \\
\hline 44 & Togo & $\begin{array}{c}\text { B. bambos, B. multiplex, B. vulgaris } \\
\text { (B. vulgaris var. striata), O. latifolia, } \\
\text { and O. abysinica. }\end{array}$ & $\begin{array}{l}\text { B. beecheyana, B. birmanica, } \\
\text { B. dissimulator, B. edulis, B. oldhamii, } \\
\text { B. nutans, B. polymorpha, B. spinosa, } \\
\text { B. ventricosa, B. warmin, D. brandisii, } \\
\text { D. latiflorus, D. membranaceus, } \\
\text { D. strictus, G. albociliata, Gigantochloa } \\
\text { bali white, Gigantochloa luteostriata, } \\
\text { Gigantochloa malay dwarf, } \\
\text { G. angustifolia, and G. chacoensis. }\end{array}$ & $\begin{array}{l}\text { Ohrnberger [1], Bystriakova } \\
\text { et al. [18], Kokutse et al. [33], } \\
\text { INBAR [17], Clayton et al. [19] }\end{array}$ \\
\hline 45 & Uganda & $\begin{array}{l}\text { H. africana, O. alpina, O. latifolia, } \\
\text { O. buchwaldii, and O. abyssinica. }\end{array}$ & D. asper & $\begin{array}{c}\text { Ohrnberger [1], Bystriakova } \\
\text { et al. [18], zhou [16], Inada and } \\
\text { Hall (2008), Ingram et al. [23], } \\
\text { INBAR [17], INBAR [34], } \\
\text { Clayton et al. [19] }\end{array}$ \\
\hline 46 & $\begin{array}{l}\text { United } \\
\text { Republic of } \\
\text { Tanzania }\end{array}$ & $\begin{array}{l}\text { B. vulgaris (B. vulgaris var. green and } \\
\text { B. vulgaris var. vittata), H. africana, } \\
\text { O. alpina, O. latifolia, O. buchwaldii, } \\
\text { and O. abyssinica. }\end{array}$ & & $\begin{array}{c}\text { Grimshaw [21], Ohrnberger [1], } \\
\text { Bystriakova et al. [18], zhou } \\
\text { [16], Inada and Hall [20], } \\
\text { INBAR [17], Clayton et al. [19] }\end{array}$ \\
\hline 47 & Zambia & $\begin{array}{l}\text { O. alpina, O. latifolia, O. buchwaldii, } \\
\text { and O. abyssinica. }\end{array}$ & & $\begin{array}{c}\text { Ohrnberger [1], Bystriakova } \\
\text { et al. [18], zhou [16], INBAR } \\
\text { [17], Clayton et al. [19] }\end{array}$ \\
\hline 48 & Zimbabwe & $\begin{array}{l}\text { O. latifolia, O. buchwaldii, and } \\
\text { O. abyssinica. }\end{array}$ & & $\begin{array}{c}\text { Ohrnberger [1], Bystriakova } \\
\text { et al. [18], INBAR [17], Clayton } \\
\text { et al. [19] }\end{array}$ \\
\hline
\end{tabular}


Table 2: A complete checklist and the scientific names of bamboo species in the African region.

\begin{tabular}{|c|c|}
\hline No. & Species name \\
\hline 1 & Bambusa balcooa Roxb. \\
\hline 2 & Bambusa bambos (L.) Voss and * Bambusa arundinacea (Retz.) Willd. \\
\hline 3 & Bambusa beecheyana Munro \\
\hline 4 & ${ }^{+}$Bambusa birmanica \\
\hline 5 & Bambusa burmanica Gamble \\
\hline 6 & Bambusa dissimulator McClure \\
\hline 7 & Bambusa emeiensis L. C. Chia and H. L. Fung \\
\hline 8 & Bambusa heterostachya (Munro) Holttum \\
\hline 9 & Bambusa lako Widjaja \\
\hline 10 & Bambusa multiplex 'Albovariegata' and * Bambusa multiplex 'Silverstripe' Fernleaf' \\
\hline 11 & $\begin{array}{c}\text { Bambusa multiplex (Lour.) Raeusch. ex Schult. f., * Bambusa multiplex f. alphonse-karrii (Mitford ex Satow) Nakai, or Bambusa } \\
\text { multiplex Roxb. }\end{array}$ \\
\hline 12 & Bambusa nutans Wall. ex Munro \\
\hline 13 & Bambusa oldhamii Munro \\
\hline 14 & Bambusa pachinensis Hayata and $*$ Bambusa textilis var. fusca McClure \\
\hline 15 & Bambusa pervariabilis McClure \\
\hline 16 & Bambusa polymorpha Munro \\
\hline 17 & Bambusa spinosa Roxb and * Bambusa blumeana Schult. f. \\
\hline 18 & Bambusa teres Munro \\
\hline 19 & Bambusa textilis McClure \\
\hline 20 & Bambusa tulda Roxb. \\
\hline 21 & Bambusa ventricosa McClure \\
\hline & Bambusa vulgaris Schrad. ex J. C. Wendl., * Bambusa madagascariensis Rivière and C. Rivière, Bambusa striata Lodd. ex Lindl., \\
\hline 22 & $\begin{array}{c}{ }^{+} \text {Bambusa vulgaris var. green, Bambusa vulgaris var. striata (Lodd. ex Lindl.) Gamble, and Bambusa vulgaris var. vittata Rivière and } \\
\text { C. Rivière }\end{array}$ \\
\hline 23 & ${ }^{+}$Bambusa warmin \\
\hline 24 & Bergbambos tessellata (Nees) Stapleton and * Thamnocalamus tessellatus (Nees) Soderstr. and R. P. Ellis \\
\hline 25 & ${ }^{+}$Brachystachyum stellatus \\
\hline 26 & Cathariostachys capitata (Kunth) S. Dransf. \\
\hline 27 & Cathariostachys madagascariensis (A. Camus) S. Dransf. \\
\hline 28 & Cephalostachyum chapelieri Munro \\
\hline 29 & Cephalostachyum perrieri A. Camus \\
\hline 30 & Cephalostachyum sp. \\
\hline 31 & Cephalostachyum viguieri A. Camus \\
\hline 32 & Dayeteng spp. \\
\hline 33 & Decaryochloa diadelpha A. Camus \\
\hline 34 & Dendrocalamus asper (Schult. Schult. f.) Backer ex K. Heyne \\
\hline 35 & Dendrocalamus barbatus Hsueh and D. Z. Li \\
\hline 36 & Dendrocalamus brandisii (Munro) Kurz and * Bambusa brandisii Munro \\
\hline 37 & Dendrocalamus giganteus Munro \\
\hline 38 & Dendrocalamus hamiltonii Nees and Arn. ex Munro \\
\hline 39 & Dendrocalamus latiflorus Munro \\
\hline 40 & Dendrocalamus longispathus (Kurz) Kurz \\
\hline 41 & Dendrocalamus membranaceus Munro \\
\hline 42 & Dendrocalamus peculiaris Hsueh and D. Z. Li \\
\hline 43 & Dendrocalamus sinicus L. C. Chia and J. L. Sun \\
\hline 44 & Dendrocalamus strictus (Roxb.) Nees \\
\hline 45 & Fargesia robusta T. P. Yi \\
\hline 46 & Gelidocalamus stellatus T. H. Wen \\
\hline 47 & Gigantochloa albociliata (Munro) Kurz \\
\hline 48 & Gigantochloa apus (Schult. f.) Kurz \\
\hline 49 & Gigantochloa atter (Hassk.) Kurz \\
\hline 50 & ${ }^{+}$Gigantochloa bali white \\
\hline 51 & Gigantochloa felix (Keng) Keng f. and * Oxytenanthera felix Keng \\
\hline 52 & Gigantochloa luteostriata Widjaja \\
\hline 53 & ${ }^{+}$Gigantochloa malay dwarf \\
\hline 54 & ${ }^{+}$Gigantochloa sumatra \\
\hline 55 & Gigantochloa verticillata (Willd.) Munro and $*{ }^{+}$Gigantochloa aff. pseudoarundinacea \\
\hline 56 & Guadua amplexifolia J. Presl in C. B. Presl \\
\hline 57 & Guadua angustifolia Kunth \\
\hline
\end{tabular}


TABLE 2: Continued.

\begin{tabular}{|c|c|}
\hline No. & Species name \\
\hline 58 & Guadua chacoensis (Rojas Acosta) Londoño and P. M. Peterson \\
\hline 59 & Guaduella densiflora Pilger ap. Engler \\
\hline 60 & Guaduella dichroa T. A. Cope \\
\hline 61 & Guaduella humilis W. D. Clayton \\
\hline 62 & Guaduella macrostachys (K. Schumann) Pilger \\
\hline 63 & Guaduella marantifolia Franchet \\
\hline 64 & Guaduella oblonga Hutchinson ex W. D. Clayton \\
\hline 65 & Hickelia africana S. Dransf. \\
\hline 66 & Hickelia alaotrensis A. Camus \\
\hline 67 & Hickelia madagascariensis A. Camus \\
\hline 68 & Hickelia perrieri (A. Camus) S. Dransf. \\
\hline 69 & Hitchcockella baronii A. Camus \\
\hline 70 & Melocanna baccifera (Roxb.) Kurz and * Melocanna bambusoides Trin. in K. P. J. Sprengel \\
\hline 71 & Nastus ambrensis A. Camus \\
\hline 72 & Nastus aristatus A. Camus \\
\hline 73 & Nastus borbonicus J. F. Gmel. \\
\hline 74 & Nastus decaryanus A. Camus \\
\hline 75 & Nastus elongatus A. Camus \\
\hline 76 & Nastus emirnensis (Baker) A. Camus \\
\hline 77 & Nastus humbertianus A. Camus \\
\hline 78 & Nastus lokohoensis A. Camus \\
\hline 79 & Nastus madagascariensis A. Camus \\
\hline 80 & Nastus manongarivensis A. Camus \\
\hline 81 & Nastus perrieri A. Camus \\
\hline 82 & Nastus tsaratananensis A. Camus \\
\hline 83 & ${ }^{+}$Nuomizhu xiaoyeteng \\
\hline 84 & Ochlandra capitata (Kunth) Camus \\
\hline 85 & Ochlandra travancorica (Bedd.) Gamble \\
\hline 86 & $\begin{array}{c}\text { Oldeania alpina (K. Schum.) Stapleton, * Arundinaria alpina K. Schum., Yushania alpina (K. Schum.) W. C. Linor, and } \\
\text { Sinarundinaria alpina (K. Schum.) C. S. Chao and Renvoize }\end{array}$ \\
\hline 87 & Olyra latifolia L. \\
\hline 88 & Oreobambos buchwaldii K. Schum. \\
\hline 89 & Oxytenanthera abyssinica (A. Rich.) Munro and * Oxytenanthera braunii Pilg. \\
\hline 90 & Perrierbambus madagascariensis A. Camus \\
\hline 91 & Perrierbambus tsarasaotrensis A. Camus \\
\hline 92 & Phyllostachys aurea (André) Rivière and C. Rivière \\
\hline 93 & $\begin{array}{c}\text { Phyllostachys edulis (Carrière) J. Houz, * Phyllostachys pubescens (Pradelle) Mazel ex J. Houz., Phyllostachys heterocycla var. } \\
\text { pubescens (Pradelle) Ohwi, or Bambusa edulis Carrière }\end{array}$ \\
\hline 94 & Phyllostachys nigra var. henonis (Mitford) Rendle \\
\hline 95 & Pleioblastus fortunei (Van Houtte) Nakai and * Sasa pygmaea (Miq.) Rehder \\
\hline 96 & Pseudosasa amabilis (McClure) Keng $\mathrm{f}$. and * Arundinaria amabilis McClure \\
\hline 97 & Pseudosasa japonica (Siebold and zucc. ex Steud.) Makino ex Nakai \\
\hline 98 & ${ }^{+}$Puelia atractocarpa \\
\hline 99 & Schizostachyum jaculans Holttum \\
\hline 100 & Schizostachyum pergracile (Munro) R. B. Majumdar in S. Karthikeyan et al. and * Cephalostachyum pergracile Munro \\
\hline 101 & Schizostachyum perrieri A. Camus \\
\hline 102 & Shibataea chinensis Nakai \\
\hline 103 & Shibataea kumasaca (Zoll. ex Steud.) Makino \\
\hline 104 & Sirochloa parvifolia (Munro) S. Dransf., * Schizostachyum parvifolium Munro, or Schizostachyum bosseri A. Camus \\
\hline 105 & Thamnocalamus ibityensis (A. Camus) Ohrnb. \\
\hline 106 & Thamnocalamus sp. \\
\hline 107 & Thyrsostachys siamensis Gamble \\
\hline 108 & Valiha diffusa S. Dransf. \\
\hline 109 & Valiha perrieri (A. Camus) S. Dransf and * Ochlandra perrieri A. Camus \\
\hline 110 & Valiha sp. \\
\hline 111 & Yushania baishazuensis Z. P. Wang and G. H. Ye \\
\hline 112 & Yushania humbertii (A. Camus) Ohrnb and * Yushania ambositrensis (A. Camus) Ohrnb. \\
\hline 113 & Yushania madagascariensis (A. Camus) Ohrnb and * Yushania marojejyensis (A. Camus) Ohrnb. \\
\hline 114 & Yushania perrieri (A. Camus) Ohrnb. \\
\hline 115 & Yushania sp. \\
\hline
\end{tabular}

Note. Most recently accepted scientific names are provided in the bamboo species checklist following Phillips [2], Ohrnberger [1], Wu et al. [3], Inada and Hall [20], INBAR [17], and Clayton et al. [19]. The most commonly used taxonomic synonyms and varieties are indicated with asterisks. Incomplete scientific names due to inadequate information are further illustrated with cross marks. 
TABLe 3: A checklist of bamboo genera classified into taxonomic tribes in the African region.

\begin{tabular}{|c|c|c|c|}
\hline \multirow{3}{*}{ No. } & \multicolumn{2}{|r|}{ Major taxonomic tribes and their respective bamboo genera in the African region } & \multirow{3}{*}{$\begin{array}{l}\text { Total number of } \\
\text { nations } \\
19\end{array}$} \\
\hline & & Tribe. Arundinarieae & \\
\hline & Genus name & Distribution of each genus in the African nations & \\
\hline 1 & Bergbambos & Lesotho & 1 \\
\hline 2 & Brachystachyum & Nigeria & 1 \\
\hline 3 & Fargesia & Nigeria & 1 \\
\hline 4 & Gelidocalamus & Nigeria & 1 \\
\hline 5 & Oldeania & $\begin{array}{l}\text { Burundi, Cameroon, Democratic Republic of Congo, Ethiopia, Kenya, Malawi, Republic of } \\
\text { Congo, Rwanda, South Sudan, Sudan, Uganda, United Republic of Tanzania, and Zambia }\end{array}$ & 13 \\
\hline 6 & Phyllostachys & Cameroon, Ethiopia, Ghana, Kenya, Madagascar, and Nigeria & 6 \\
\hline 7 & Pleioblastus & Nigeria & 1 \\
\hline 8 & Pseudosasa & Algeria, Morocco and Kenya & 3 \\
\hline 9 & Shibataea & Kenya and Nigeria & 2 \\
\hline 10 & Thamnocalamus & Lesotho and Madagascar & 2 \\
\hline \multirow[t]{2}{*}{11} & Yushania & Madagascar & 1 \\
\hline & & Tribe. Bambuseae & 45 \\
\hline 12 & Bambusa & $\begin{array}{c}\text { Benin, Burkina Faso, Cameroon, Cote d'Ivoire, Democratic Republic of Congo, Egypt, } \\
\text { Ethiopia, Ghana, Guinea, Kenya, Libya, Madagascar, Mozambique, Mauritius, Mozambique, } \\
\text { Nigeria, Rwanda, São Tomé and Príncipe, Sierra Leone, Seychelles, South Africa, Sudan, } \\
\text { Togo, and United Republic of Tanzania }\end{array}$ & 24 \\
\hline 13 & Cathariostachys & Madagascar & 1 \\
\hline 14 & Cephalostachyum & Madagascar & 1 \\
\hline 15 & Decaryochloa & Madagascar & 1 \\
\hline 16 & Dendrocalamus & $\begin{array}{l}\text { Benin, Democratic Republic of Congo, Ethiopia, Ghana, Kenya, Madagascar, Mauritius, } \\
\text { Mozambique, Nigeria, Réunion, Seychelles, Sudan, Togo, and Uganda }\end{array}$ & 14 \\
\hline 17 & Gigantochloa & Ethiopia, Ghana, Madagascar, and Togo & 4 \\
\hline 18 & Guadua & Ethiopia, Ghana, and Uganda & 3 \\
\hline 19 & Hickelia & Kenya, Madagascar, Uganda, and United Republic of Tanzania & 4 \\
\hline 20 & Hitchcockella & Madagascar & 1 \\
\hline 21 & Melocanna & Sudan & 1 \\
\hline 22 & Nastus & Madagascar & 1 \\
\hline 23 & Ochlandra & Madagascar & 1 \\
\hline 24 & Oreobambos & $\begin{array}{l}\text { Angola, Burundi, Democratic Republic of Congo, Kenya, Malawi, Mozambique, Uganda, } \\
\text { United Republic of Tanzania, Zambia, and Zimbabwe }\end{array}$ & 10 \\
\hline 25 & Oxytenanthera & $\begin{array}{l}\text { Angola, Benin, Burkina Faso, Burundi, Cameroon, Central African Republic, Chad, Cote } \\
\text { d'Ivoire, Democratic Republic of Congo, Equatorial Guinea, Eritrea, Ethiopia, Gambia, } \\
\text { Ghana, Guinea, Guinea-Bissau, Kenya, Lesotho, Liberia, Malawi, Mali, Mozambique, Niger, } \\
\text { Nigeria, Republic of Congo, Rwanda, São Tomé and Príncipe, Senegal, Sierra Leone, South } \\
\text { Africa, South Sudan, Sudan, Swaziland, Togo, Uganda, United Republic of Tanzania, Zambia, } \\
\text { and Zimbabwe }\end{array}$ & 38 \\
\hline 26 & Perrierbambus & Madagascar & 1 \\
\hline 27 & Schizostachyum & Ethiopia, Kenya, and Madagascar & 3 \\
\hline 28 & Sirochloa & Comoros and Madagascar & 2 \\
\hline 29 & Thyrsostachys & Ethiopia, Ghana, and Kenya & 3 \\
\hline \multirow[t]{2}{*}{30} & Valiha & Madagascar & 1 \\
\hline & & Tribe. Guaduelleae & 10 \\
\hline \multirow[t]{2}{*}{31} & Guaduella & $\begin{array}{c}\text { Angola, Cameroon, Cote d'Ivoire, Gabon, Ghana, Guinea, Liberia, Nigeria, Republic of } \\
\text { Congo, and Sierra Leone }\end{array}$ & 10 \\
\hline & & Tribe. Olyreae & 30 \\
\hline 32 & Olyra & $\begin{array}{c}\text { Angola, Benin, Burkina Faso, Burundi, Cameroon, Central African Republic, Comoros, Cote } \\
\text { d'Ivoire, Democratic Republic of Congo, Equatorial Guinea, Ethiopia, Gambia, Ghana, } \\
\text { Guinea, Guinea-Bissau, Kenya, Liberia, Madagascar, Malawi, Nigeria, Republic of Congo, } \\
\text { São Tomé and Príncipe, Senegal, Sierra Leone, Sudan, Togo, Uganda, United Republic of } \\
\text { Tanzania, Zambia, and Zimbabwe }\end{array}$ & 30 \\
\hline \multirow{3}{*}{33} & & Tribe. Puelieae & 1 \\
\hline & Puelia & Cameroon & 1 \\
\hline & & Tribe. Others & 1 \\
\hline 34 & Dayeteng & Nigeria & 1 \\
\hline 35 & Nuomizhu & Nigeria & 1 \\
\hline
\end{tabular}

Note. Listed bamboo genera are classified into each taxonomic tribe following Ohrnberger [1], Wu et al. [3], Inada and Hall [20], INBAR [17], and Clayton et al. [19]. 
TABLE 4: A summary of bamboo resource diversity and distribution in the African region.

\begin{tabular}{|c|c|c|c|c|c|c|c|c|c|c|}
\hline \multirow{2}{*}{$\begin{array}{l}\text { African } \\
\text { region }\end{array}$} & \multicolumn{5}{|c|}{ Species } & \multicolumn{5}{|c|}{ Genera } \\
\hline & Indigenous & & Introduced & & Total & Indigenous & & Introduced & & Total \\
\hline $\begin{array}{l}\text { Mainland } \\
\text { Africa }\end{array}$ & $\begin{array}{l}\text { B. bambos, B. multiplex, } \\
\text { B. pervariabilis, } \\
\text { B. vulgaris, B. tessellata, } \\
\text { D. strictus, G. felix, } \\
\text { G. densiflora, G. dichroa, } \\
\text { G. humilis, } \\
\text { G. macrostachys, } \\
\text { G. marantifolia, } \\
\text { G. oblonga, H. africana, } \\
\text { O. alpina, O. latifolia, } \\
\text { O. buchwaldii, } \\
\text { O. abyssinica, } \\
\text { P. amabilis, } \\
\text { P. atractocarpa, and } \\
\text { Thamnocalamus sp. }\end{array}$ & 21 & 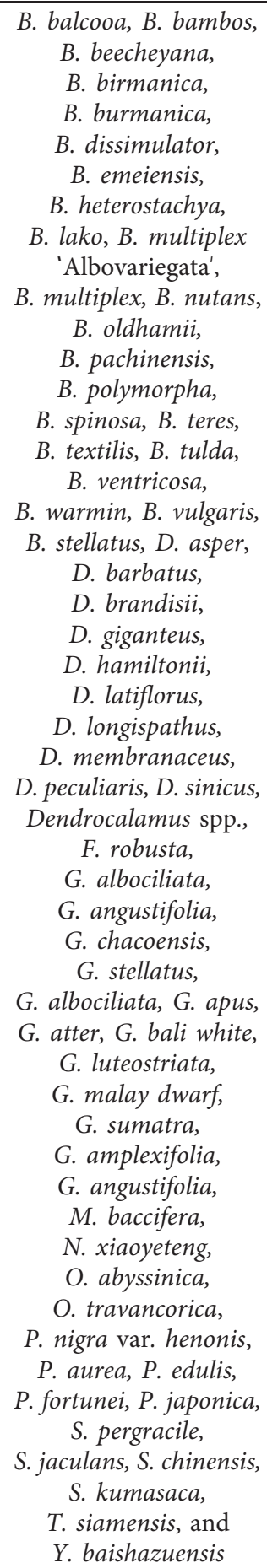 & 63 & 78 & $\begin{array}{c}\text { Bambusa, } \\
\text { Bergbambos, } \\
\text { Dendrocalamus, } \\
\text { Gigantochloa, } \\
\text { Guaduella, Hickelia, } \\
\text { Oldeania, Olyra, } \\
\text { Oreobambos, } \\
\text { Oxytenanthera, } \\
\text { Pseudosasa, Puelia, } \\
\text { and Thamnocalamus }\end{array}$ & 13 & $\begin{array}{c}\text { Bambusa, } \\
\text { Brachystachyum, } \\
\text { Dayeteng, } \\
\text { Dendrocalamus, } \\
\text { Fargesia, } \\
\text { Gelidocalamus, } \\
\text { Gigantochloa, } \\
\text { Guadua, } \\
\text { Melocanna, } \\
\text { Nuomizhu, } \\
\text { Ochlandra, } \\
\text { Oxytenanthera, } \\
\text { Phyllostachys, } \\
\text { Pleioblastus, } \\
\text { Pseudosasa, } \\
\text { Schizostachyum, } \\
\text { Shibataea, } \\
\text { Thyrsostachys, and } \\
\text { Yushania }\end{array}$ & 19 & 27 \\
\hline
\end{tabular}


TABLE 4: Continued.

\begin{tabular}{|c|c|c|c|c|c|c|c|c|c|c|}
\hline \multirow{2}{*}{$\begin{array}{l}\text { African } \\
\text { region }\end{array}$} & \multicolumn{5}{|c|}{ Species } & \multicolumn{5}{|c|}{ Genera } \\
\hline & Indigenous & & Introduced & & Total & Indigenous & & Introduced & & Total \\
\hline Six islands & $\begin{array}{l}\text { B. stellatus, C. capitata, } \\
\text { C. madagascariensis, } \\
\text { C. chapelieri, C. perrieri, } \\
\text { Cephalostachyum. sp., } \\
\text { C. viguieri, D. diadelpha, } \\
\text { H. alaotrensis, } \\
\text { H. madagascariensis, } \\
\text { H. perrieri, H. baronii, } \\
\text { N. aristatus, } \\
\text { N. borbonicus, } \\
\text { N. decaryanus, } \\
\text { N. elongatus, } \\
\text { N. emirnensis, } \\
\text { N. humbertianus, } \\
\text { N. lokohoensis, } \\
\text { N. madagascariensis, } \\
\text { N. manongarivensis, } \\
\text { N. perrieri, } \\
\text { N. tsaratananensis, } \\
\text { N. ambrensis, } \\
\text { O. abyssinica, } \\
\text { O. capitata, O. latifolia, } \\
\text { P. madagascariensis, } \\
\text { P. tsarasaotrensis, } \\
\text { S. perrieri, S. parvifolia, } \\
\text { T. ibityensis, } \\
\text { Thamnocalamus sp., } \\
\text { V. perrieri, V. diffusa, } \\
\text { Valiha sp.,Y. humbertii, } \\
\text { Y. madagascariensis, } \\
\text { Y. perrieri, and } \\
\text { Yushania sp. } \\
\text { Totl }\end{array}$ & 40 & $\begin{array}{c}\text { B. balcooa, } \\
\text { B. multiplex, B. spinosa, } \\
\text { B. vulgaris, D. asper, } \\
\text { D. giganteus, } \\
\text { D. strictus, G. aff. } \\
\text { Pseudoarundinacea, } \\
\text { P. aurea, and P. nigra }\end{array}$ & 10 & 50 & $\begin{array}{c}\text { Brachystachyum, } \\
\text { Cathariostachys, } \\
\text { Cephalostachyum, } \\
\text { Decaryochloa, } \\
\text { Hickelia, } \\
\text { Hitchcockella, } \\
\text { Nastus, Ochlandra, } \\
\text { Olyra, } \\
\text { Oxytenanthera, } \\
\text { Perrierbambus, } \\
\text { Schizostachyum, } \\
\text { Sirochloa, } \\
\text { Thamnocalamus, } \\
\text { Valiha, and Yushania }\end{array}$ & 16 & $\begin{array}{c}\text { Bambusa, } \\
\text { Dendrocalamus, } \\
\text { Gigantochloa, and } \\
\text { Phyllostachys }\end{array}$ & 4 & 20 \\
\hline
\end{tabular}

back within the region. In this case, O. abyssinica is well known among 38 (79.2\%) African nations, followed by O. alpina with a total of $13(27.1 \%)$ African countries. On the other hand, $27.1 \%$ of the nations contain both species. For example, their total area coverage only from Ethiopia is 1.44 million ha [35], suggesting that a huge resource potential is found in the region. Their tremendous socioeconomic, cultural, and ecological uses commonly practiced by the local people are also cited as a model for bamboo resource utilization. Among others, Ethiopia is well-known for the untapped resource potential and wider distribution of these species in different agroecologies. There are also relatively more previous works carried out, and better information is comparatively available for these species. With this understanding, detail literature review on general background, biology, origin and distribution, status and resource potential, multipurpose uses, and silvicultural applications of $O$. abyssinica and O. alpina are extensively conducted and provided from Ethiopia. Figures and photos are further provided by the corresponding author from his previous professional experience in forestry research at the national research system particularly for indigenous bamboo species.

\subsection{Oldeania alpina (K. Schum.)}

Common name: highland/alpine/African alpine bamboo $[2,4]$

Local name: Kerkeha in Amharic and Lemen in Affan Oromo languages [2, 39].

Synonymous: Arundinaria alpina K. Schum., Yushania alpina (K. Schum.) W. C. Lin, and Sinarundinaria alpina (K. Schum.) Chao and Renv [2, 4, 17]

Description: it grows up to a maximum height of $17 \mathrm{~m}$ and diameter of $13 \mathrm{~cm}$ from a stout branching rhizome [4] 
TABLE 5: A summary of the origin and distribution of bamboo resource in the African region.

\begin{tabular}{|c|c|c|c|}
\hline \multirow{2}{*}{ List of countries } & \multicolumn{3}{|c|}{ Origin of bamboo species } \\
\hline & Indigenous & Introduced & Total \\
\hline Algeria & & 1 & 1 \\
\hline Angola & 5 & & 5 \\
\hline Benin & 2 & 3 & 5 \\
\hline Burkina Faso & 2 & 1 & 3 \\
\hline Burundi & 4 & & 4 \\
\hline Cameroon & 10 & 3 & 13 \\
\hline Central African Republic & 2 & & 2 \\
\hline Chad & 1 & & 1 \\
\hline Comoros* & 2 & & 2 \\
\hline Cote d'Ivoire & 3 & 1 & 4 \\
\hline Democratic Republic of Congo & 4 & 2 & 6 \\
\hline Egypt & & 2 & 2 \\
\hline Equatorial Guinea & 2 & & 2 \\
\hline Eritrea & 1 & & 1 \\
\hline Ethiopia & 3 & 25 & 28 \\
\hline Gabon & 3 & & 3 \\
\hline Gambia & 2 & & 2 \\
\hline Ghana & 8 & 16 & 24 \\
\hline Guinea & 2 & 1 & 3 \\
\hline Guinea-Bissau & 2 & & 2 \\
\hline Kenya & 5 & 16 & 21 \\
\hline Lesotho & 3 & & 3 \\
\hline Liberia & 3 & & 3 \\
\hline \multirow[t]{2}{*}{ Libya } & & 1 & 1 \\
\hline & Indigenous & Introduced & Total \\
\hline Madagascar* & 37 & 8 & 45 \\
\hline Malawi & 4 & & 4 \\
\hline Mali & 1 & & 1 \\
\hline Mauritius* & 1 & 2 & 3 \\
\hline Morocco & & 1 & 1 \\
\hline Mozambique & 3 & 4 & 7 \\
\hline Niger & 1 & & 1 \\
\hline Nigeria & 4 & 12 & 16 \\
\hline Republic of Congo & 4 & & 4 \\
\hline Réunion * & 1 & 1 & 2 \\
\hline Rwanda & 2 & 1 & 3 \\
\hline São Tomé and Príncipe ${ }^{*}$ & 2 & 2 & 4 \\
\hline Senegal & 2 & & 2 \\
\hline Seychelles* & & 5 & 5 \\
\hline Sierra Leone & 3 & 1 & 4 \\
\hline South Africa & 2 & 1 & 3 \\
\hline South Sudan & 2 & & 2 \\
\hline Sudan & 3 & 10 & 13 \\
\hline Swaziland & 1 & & 1 \\
\hline United Republic of Tanzania & 6 & & 6 \\
\hline Togo & 5 & 20 & 25 \\
\hline Uganda & 5 & 1 & 6 \\
\hline Zambia & 4 & & 4 \\
\hline Zimbabwe & 3 & & 3 \\
\hline
\end{tabular}

Note. The six islands surrounding the mainland Africa are indicated with asterisks. The species list for each country is in Table 1.

Rhizome type: there is a controversial issue on the rhizome type of $O$. alpina (Figure 2(a)). It is either monopodial or leptomorph rhizome type [2] or pachymorph or sympodial rhizome type $[4,29]$. According to Meredith [4], some of the rhizome necks are exceptionally elongated and exhibit a spreading habit instead of forming a dense clump [4]. Such loose clump-forming pachymorph rhizome makes the species improperly considered under the running or creeping rhizome type, i.e., monopodial rhizome type [29].

Culms sheaths: the culm sheath (Figure 4(a)), which is covered with dense hairs, contains reddish-brown bristles and fimbriate auricles at the tip part [2] 
TABle 6: Status and potential of bamboo resource in the African region.

\begin{tabular}{|c|c|c|c|c|c|c|c|}
\hline Country & $\begin{array}{l}\text { Bamboo area } \\
(1000 \mathrm{ha})\end{array}$ & $\begin{array}{l}\text { Bamboo } \\
\text { area }(\%)\end{array}$ & $\begin{array}{c}\text { Forest area } \\
\text { (1000 ha), GFRA } \\
(2015)\end{array}$ & $\begin{array}{c}\text { Bamboo to } \\
\text { forest area } \\
(\%)\end{array}$ & $\begin{array}{c}\text { Year of } \\
\text { available } \\
\text { data }\end{array}$ & Reference & Remark \\
\hline Cameroon & 5 & 0.11 & 18816 & 0.03 & 2010 & $\begin{array}{l}\text { Ingram et al. } \\
{[23]}\end{array}$ & $\begin{array}{l}\text { Data only from } \\
\text { northwest of } \\
\text { Cameroon. }\end{array}$ \\
\hline Congo & 102 & 2.24 & 22334 & 0.46 & 1988 & $\begin{array}{l}\text { UNDIO }[39,42] \\
\text { cited in FAO [7] }\end{array}$ & \multirow{12}{*}{$\begin{array}{c}\text { Overestimated and not } \\
\text { verified. }\end{array}$} \\
\hline Ethiopia & 1439 & 31.55 & 12499 & 11.51 & 2018 & Zhao et al. [35] & \\
\hline Ghana & 400 & 8.77 & 9337 & 4.28 & 2015 & INBAR [27] & \\
\hline Kenya & 131 & 2.87 & 4413 & 2.97 & 2018 & Zhao et al. [35] & \\
\hline Nigeria & 1590 & 34.86 & 6993 & 22.74 & 2007 & FAO [7] & \\
\hline Rwanda & 17 & 0.37 & 480 & 3.54 & 1985 & $\begin{array}{c}\text { FRA (1985) cited } \\
\text { in FAO [7] }\end{array}$ & \\
\hline Senegal & 661 & 14.49 & 8273 & 7.99 & 2010 & FAO $[36]$ & \\
\hline Sudan & 31 & 0.68 & 19210 & 0.16 & 2010 & FAO [36] & \\
\hline Uganda & 54.6 & 1.20 & 2077 & 2.63 & 2018 & Zhao et al. [35] & \\
\hline $\begin{array}{l}\text { United } \\
\text { Republic of } \\
\text { Tanzania }\end{array}$ & 128 & 2.81 & 46060 & 0.28 & 2010 & $\begin{array}{l}\text { FAO [7] cited in } \\
\text { FAO [36] }\end{array}$ & \\
\hline Zimbabwe & 3.2 & 0.07 & 14062 & 0.02 & 2001 & $\begin{array}{c}\text { FAO (2001) } \\
\text { cited in FAO [7] }\end{array}$ & \\
\hline Total & 4561.8 & 100 & 164554 & 100 & & & \\
\hline
\end{tabular}
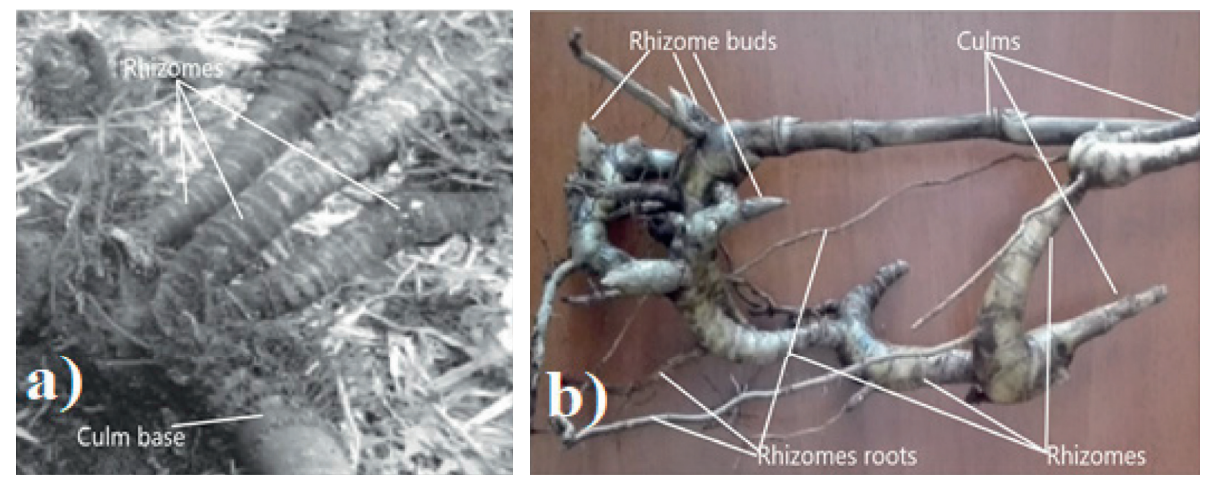

FIgURE 2: Rhizome type of (a) O. alpina (source: [37]) and (b) O. abyssinica. Clumps and culms: it is characterized by erect, thick-walled, and hollow culm bamboo species (Figures 3(a) and 3(b)).

Flowering pattern: the flowering pattern of O. alpina (Figure 5) is sporadic flowering [29, 39]. This means only some individuals or clumps within the bamboo forest are flowered, produce seeds, and eventually died, while the rest part of the bamboo forest is alive [39]. Yet, Kigomo [29] reported that after the flowering of the species, seeds are produced and still the flowered clumps are alive instead of dying.

Inflorescence: the paniculate inflorescence is loose to fairly compact in appearance. The shape of the spikelet, which is comprised 4-11 flowers, ranged from linear to linear-elliptic [2]. The author also noted that lanceolate to oblong-shaped lemmas on each spikelet are covered with hairs.

Distribution: O. alpina is found in montane forest often on volcanic soils, with Podocarpus in upland rainforest and with Juniperus in drier forest frequently planted along roads and in villages [2]. The species, which is indigenous to equatorial Africa, can grow in full sunlight but can also be found within a minimum temperature of $-4 \mathrm{C}$ [4]. It is distributed in Gojam, Shewa, Kefa, Gamo Gofa, Sidamo, and Bale regions (Figure 6) at the altitudes ranging from $2200 \mathrm{~m}$ to $4000 \mathrm{~m}$ above the sea level [2].

Silvicultural application: despite the limited availability of seeds, the species is propagated by seeds as shown in Figure 7(a) [40] or collected seedlings from the wild at nursery. The species is also vegetatively propagated through offset cutting, culm cutting [29, 41], culm layering, branch cutting, rhizome cutting [41], and macroproliferation $[29,39]$. 

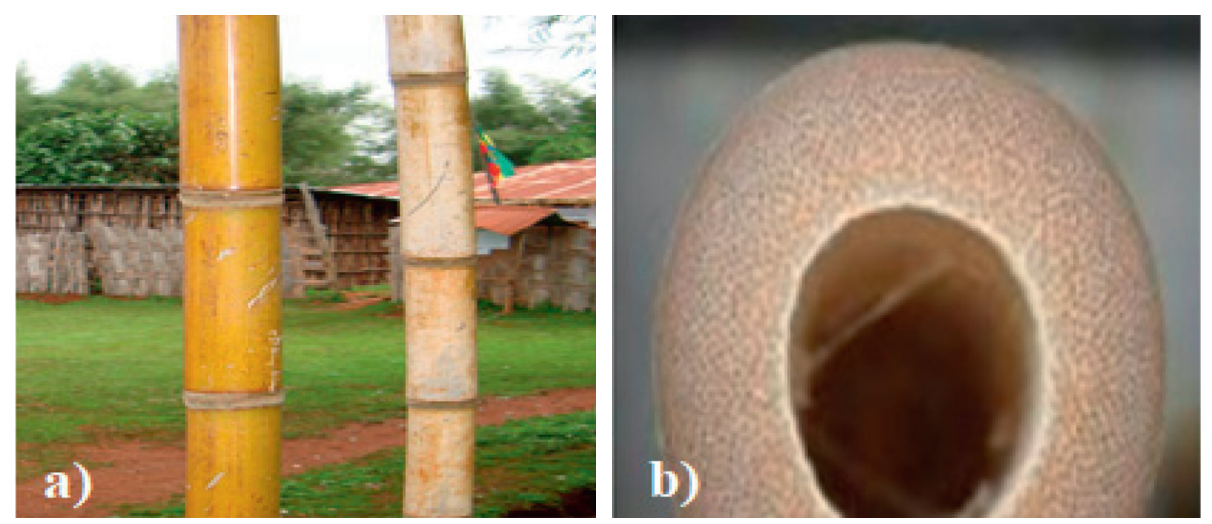

Figure 3: O. alpina standing culms (a) and thick and hollow culm (b).
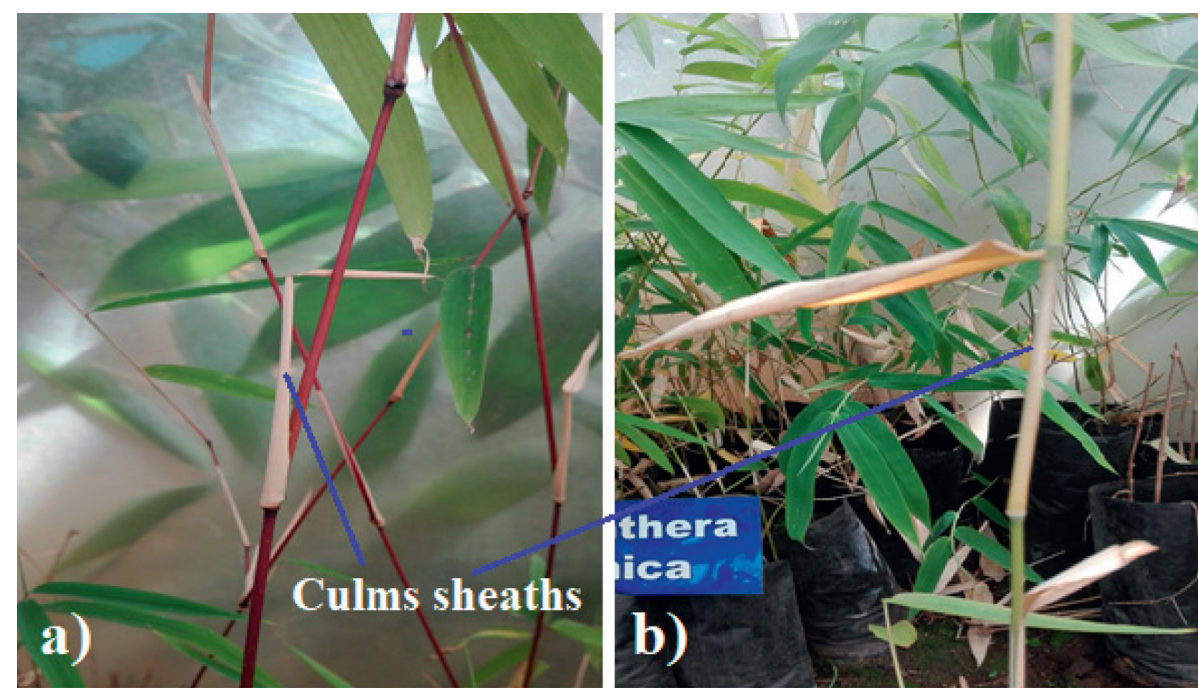

Figure 4: Culms sheaths of (a) O. alpina and (b) O. abyssinica. Leaves: linear lanceolate-shaped leaf blade is extended from the culm sheath.
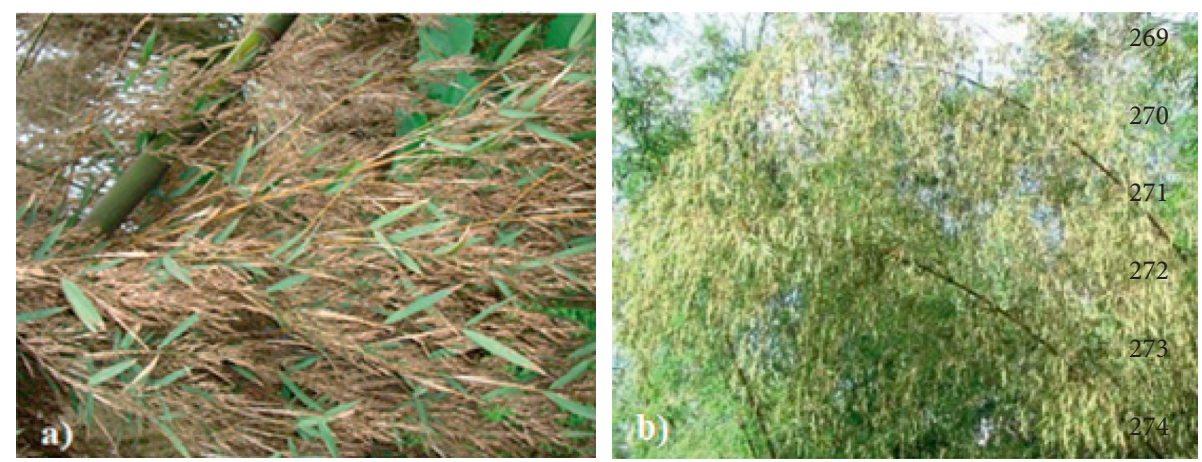

Figure 5: Bamboo mass flowering and seed production of (a) O. alpina in Hula district of Sidama Zone, SNNPR in 2017 and (b) O. abyssinica (source: [38]).

Once seedlings are raised at nursery or green house (Figure 8(a)), weeding, hoeing, fertilizer application, supervision of insect and pest, and acclimatization (hardening) are carried out. Following this, seedlings are safely transported to prepared plantation sites and planted with or without the application of organic manure. Once established, the survival rate and growth performance of seedlings are supervised and dead seedlings are replaced. Hereafter, various plantation managements including thinning, fertilizer application, selective cutting, regular weeding and cleaning, soil loosening, supervision of insect pests and diseases, and controlling animal browsing, rodent damage, and fire outbreaks are conducted. 


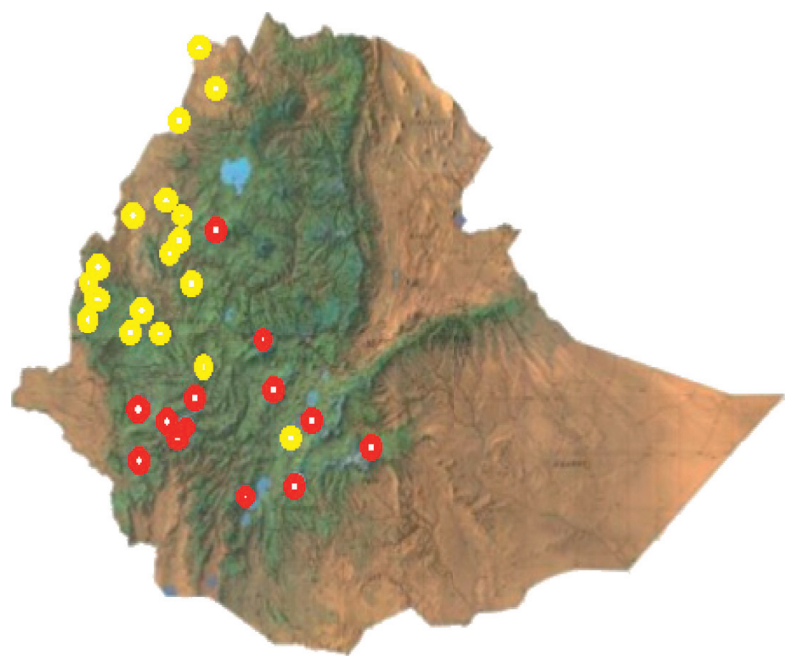

Key

- Highland bamboo

Lowland bamboo

FIGURE 6: Geographical distribution of O. alpina and O. abyssinica in Ethiopia. Origin: indigenous to Ethiopia and endemic to Africa $[13,14]$.
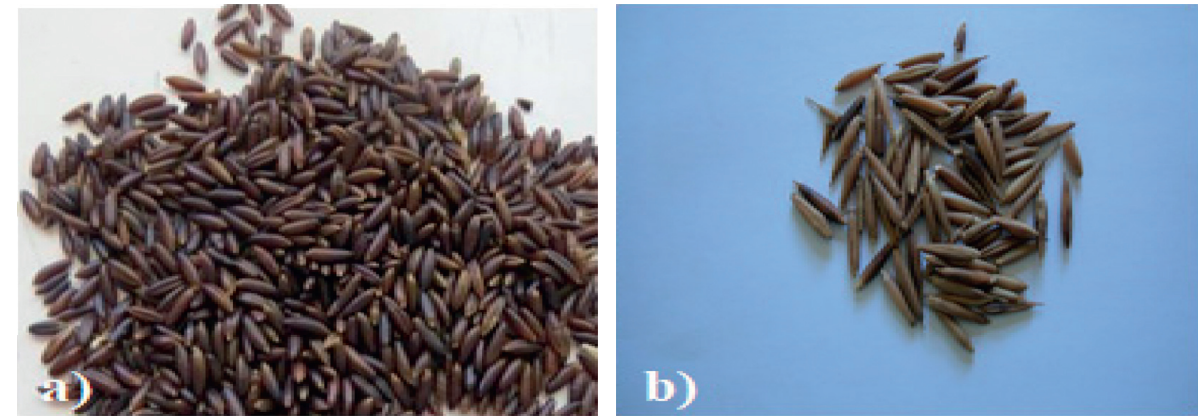

Figure 7: Fresh collected seeds of (a) O. alpina from Hula district and (b) O. abyssinica from Assosa district after mass flowering and fruiting in 2017.
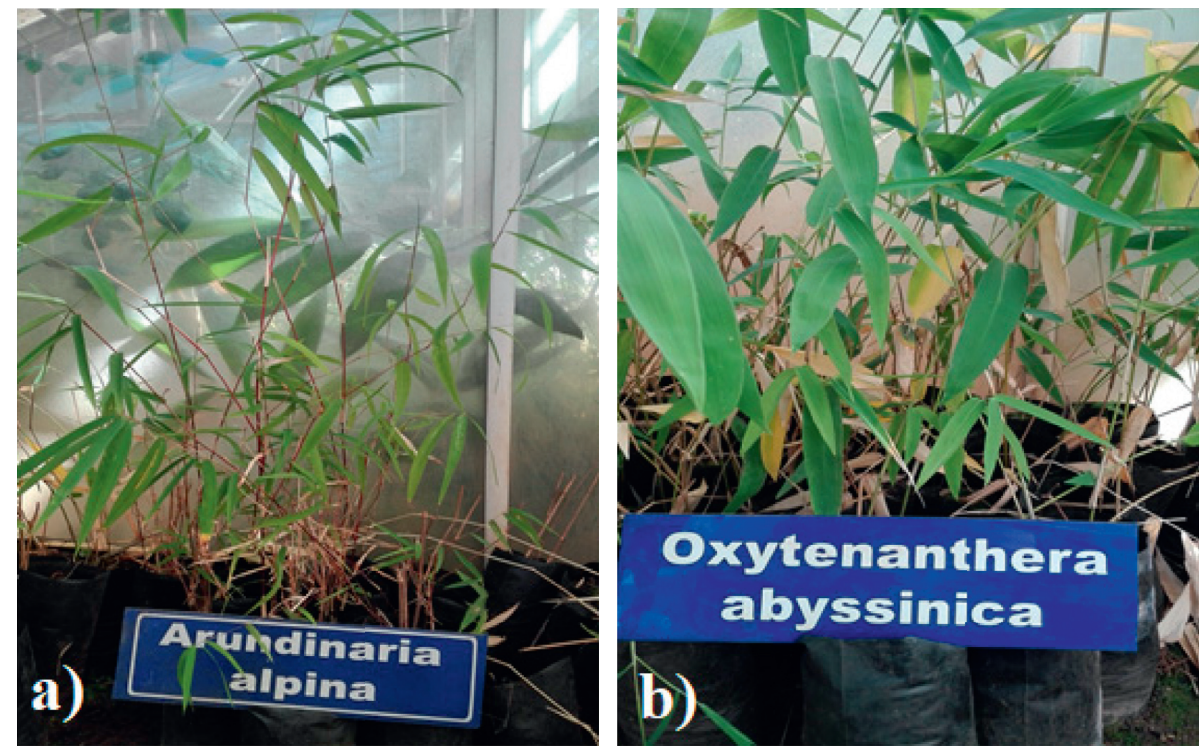

FIgURE 8: O. alpina (a) and O. abyssinica (b) seedlings at CEE-FRC greenhouse in January 2021, Addis Ababa. 


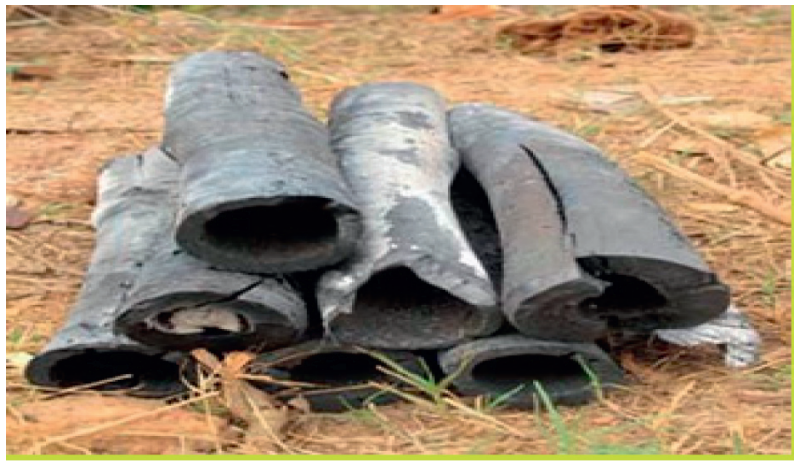

Figure 9: Bamboo charcoal making in Ethiopia (source: [22]).
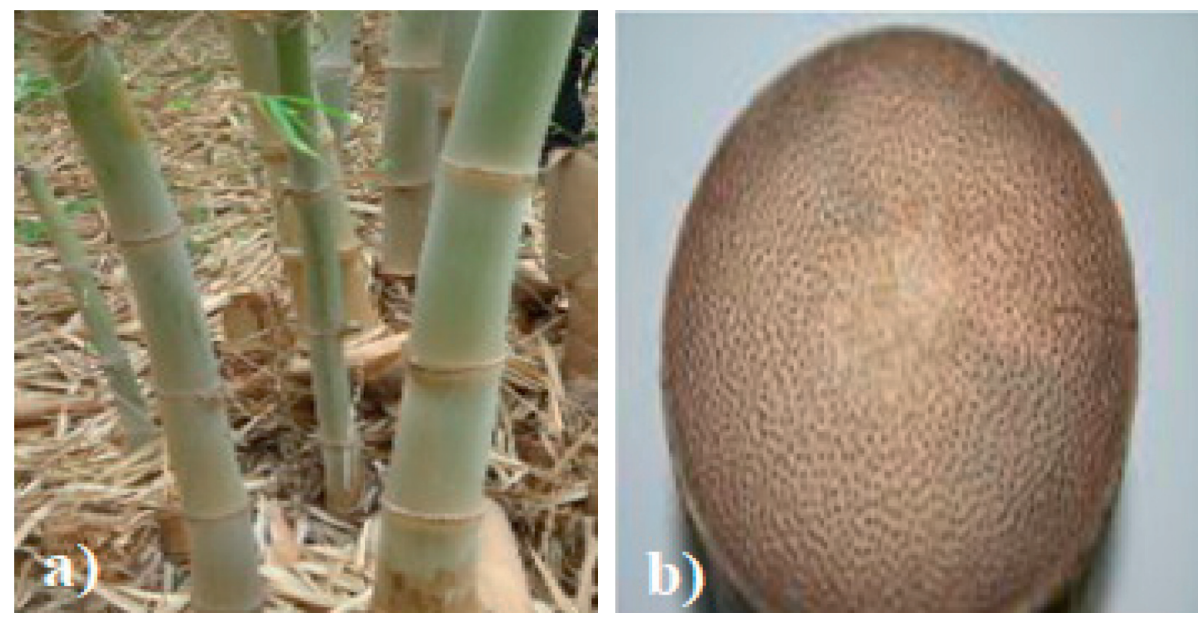

FIgURE 10: O. abyssinica standing culms (a) and thick and solid culms after maturation (b).
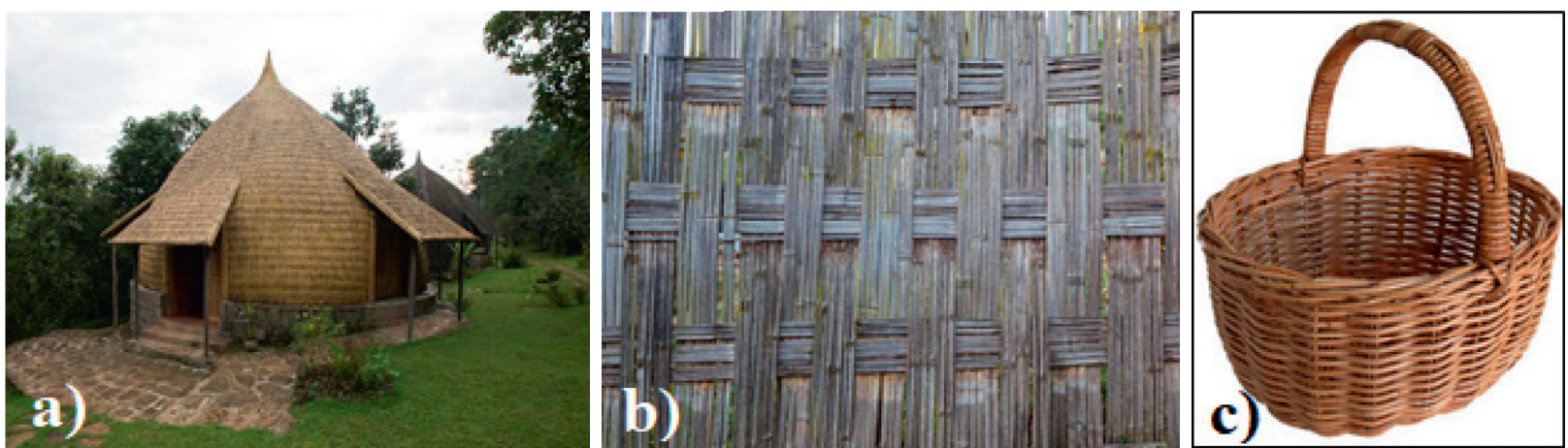

Figure 11: Traditional use of bamboo for (a) house construction, (b) fencing, and (c) household utensils (e.g., basket).

Uses: traditional house construction and fencing, furniture and household utensils, farming tools, livestock fodder and traditional medicine, production of handicrafts (basketry, mats, hats, and decorative items), water pipes, weaving, beehive, musical instruments and weapons, walking sticks, furniture, and other household utensils. In recent years, various industries and factories are emerged on processing and production of bamboo products. Some of these products are production of toothpicks and chopsticks, parquet flooring, window blinds, curtains, bioenergy (charcoal and briquettes (Figure 9)), and other related products for local and international market.

\subsection{Oxytenanthera abyssinica (A. Rich.) Munro}

Common name: lowland bamboo [2, 13, 14, 42]

Local name: Shimel in Amharic and Shimalla in Affan Oromo [2, 42]

Synonymous: Bambusa abyssinica A. Rich. [2, 13, 14] Description: O. abyssinica grows up to a height of $13 \mathrm{~m}$ and a diameter of $10 \mathrm{~cm} \mathrm{[2]}$

Rhizome type: the species is a solid clump-forming bamboo and classified under the sympodial or pachymorph rhizome type $[2,4,29,42]$. It is 
characterized by a short-necked rhizome type, and each new rhizome turns up ward and sprouts to shoot and eventually develops into a young culm (Figure 2(b)).

Culms: it has an erect or ascending culm with a height of 3-13 $\mathrm{m}$ and a culm diameter of $5-10 \mathrm{~cm}$ [2]. Unlike most of the bamboo species, it has semisolid culm (Figures 10(a) and 10(b)) during the early stage of development but solid after maturation [2, 4, 29, 42]. During the early young stage, the culm is silky and hairy, while shiny with various colors after maturation [2].

Culms sheaths: it is covered with brown hairs with leaf blade at its tip part (Figure 4(a)). However, the sheath further consists of a few deciduous setae on the shoulders but without auricles [2].

Leaves: narrow lanceolate-oblong leaf blade is attached to culm sheath with a very short petiole or pseudopetiole [2]

Flowering pattern: the flowering pattern of O. abyssinica is gregarious flowering (Figure 5(b)), i.e., mass flowering followed by mass death of the whole bamboo forest after seed production [38, 42]. Still other findings report that the species has both flowering patterns, i.e., cyclical gregarious flowering and unpredictable sporadic flowering [29, 42]. This suggests that there is no consistency of data on the flowering intervals of these species, and it varies among different sources. Therefore, detail and long-term study on flowering phenomena needs special attention.

Inflorescence: this species has an inflorescence with a spiky globose head and characterized by narrow lanceolate-shaped spikelet [2]. In addition, the same author noted that the fertile lemmas have comparable length to the spikelet unlike the sterile lemmas with a shorter size.

Distribution: the species is widely distributed in lowland regions of western and northwestern parts of Ethiopia [43] in Tigray, Gonder, Gojam, and Welega regions as shown in Figure 6 [2]. Oxytenanthera abyssinica is found in savanna woodland, favoring river valleys, often forming extensive stands at the altitudes ranging from $1200 \mathrm{~m}$ to $1800 \mathrm{~m}$ above the sea level [2]. Yet, Meredith [4] explained that it is distributed throughout tropical Africa at altitudinal ranges from near the sea level to $2000 \mathrm{~m}$ in savannahs and on hillsides. Its annual rainfall ranges $700-1000 \mathrm{~mm}$, which is concentrated over a period of three to four months with the mean annual temperature of above $30 \mathrm{C}$ [42]. The species can grow at a minimum temperature of $-1 \mathrm{C}$ and prefers moist conditions along waterways [4]. However, this author further found out that $O$. abyssinica can grow in full sunlight, and it is also drought resistant and may be deciduous in hot and dry conditions. The species is easily adaptable to poor soils and provided as a buffer zone for desert areas. The species form either a large area pure forest or they are found as middle and lower layers in the mixed forest associated by other species in the mountainous areas.
Origin: indigenous to Ethiopia and endemic to Africa $[13,14]$

Silvicultural application: the species is propagated by seeds as shown in Figure 7(b) [29, 40, 44-47] or collected seedlings from the wild at nursery. There is also better availability of $O$. abyssinica seeds, higher seed germination, and better greenhouse performance of seedlings compared to O. alpina (Figure 8)(b) [40]. In addition, the species is vegetatively propagated through offset cutting, macroproliferation [29, 42], and tissue culture [48]. Once seedlings are raised at nursery or green house, weeding, hoeing, fertilizer application, supervision of insects and pests, and acclimatization (hardening) are carried out [46]. Following this, seedlings are safely transported to prepared plantation sites and planted with or without the application of organic manure. Once established, the survival rate and growth performance of seedlings are supervised and dead seedlings are replaced. Hereafter, various plantation managements including thinning, fertilizer application, selective cutting, regular weeding and cleaning, soil loosening, supervision of insects, pests, and diseases, and controlling animal browsing, rodent damage, fire outbreaks are conducted.

Uses: traditional house construction and fencing, furniture and household utensils, farming tools, edible shoot production, livestock fodder and traditional medicine, production of handicrafts (basketry, mats, hats, and decorative items), water pipes, weaving, beehive, musical instruments and weapons, walking sticks, furniture, and other household utensils. In recent years, various industries and factories are emerged on processing and production of bamboo products. Some of these products are production of toothpicks and chopsticks, parquet flooring, window blinds, curtains, bioenergy (charcoal and briquette), and other related products for local and international market.

\section{Opportunities and Challenges on Bamboo Resource in the African Region}

6.1. Opportunities. Our extensive literature review indicated that bamboo resource has some tremendous opportunities for its development and promotion in the region. The African region has untapped bamboo resource potential with immense socioeconomic, cultural, and ecological significances to local people (Figures 11(a)-11(c)). At the same time, due to its fast growth rate, high biomass production, and short rotation period, bamboo resource is one of the most promising and suitable species to replace the forest resource. In recent years, emerging community-based bamboo processing cooperatives, enterprises, and industries for different end products are some of the opportunities. Some of these products are production of baskets, mats, toothpicks and chopsticks, parquet flooring, window blinds, curtains, and other related products for local and international market. Bamboo biomass also can provide a sustained source of feedstock for bamboo-based bioenergy 
production. Thus, bioenergy is produced by the conversion of bamboo biomass into solid fuels (firewood, charcoal, and briquette for cooking, heating, and lighting), liquid fuels or biofuels (bioethanol and biomethane production), and biogas (to produce power or electricity) [49]. Its suitability to replace the role of forest products such as timber and wood is its best potential value. For instance, bamboo culms are commonly served as scaffoldings and replaced the role of iron steel or wood functions [50]. Nowadays, timber harvesting, bamboo poles, and scaffoldings are emerging opportunities for bamboo resource development. Selling of bamboo culms is one of the attractive income generating activities in the bamboo sector in Ethiopia. According to Lou [8], the global bamboo trade is estimated to be between $\$ 1.5$ and 2.5 billion. Out of $\$ 18$ million exports of bamboo products in African, Ethiopia contributes $\$ 0.23$ million, which is accounted for about $0.02 \%$ of the global exports [51]. The same study also reported that bamboo pole, which is the most exported bamboo commodity, accounted for about $\$ 0.23$ million. A total of 89,845 highland bamboo poles are produced by the smallholder households for house construction, furniture production, handcraft making (bed, table, chair, shelve, and mat), fencing, and household utensils [52]. In turn, the average total annual income from bamboo in Sidama, Awi, and Sheka is 2235, 2084, and 284 Birr, respectively [53]. In the same way, out of the average annual 21000 bamboo culms supply, an average annual $\$ 6738$ net income is earned at Addis Ababa market [54]. Edible bamboo shoots are one of the most important sources of daily dish with rich sources of nutritional contents. For instance, the nutritional analysis of indigenous bamboo species in Ethiopia (A. alpina and O. abyssinica) indicated that both species have almost comparable moisture content, ash, crude fiber, protein, fat, and mineral (iron, zinc, and sodium) composition [55]. By contrast, the same authors found that tannin and phytate contents in O. abyssinica and $\mathrm{HCN}$ in A. alpina are low. Thus, bamboo shoots production is one of the most promising species to ensure food security especially in the rural setting. Associated with this resource base assessment, introducing new species from elsewhere, propagation, utilization, and management practices of the bamboo resources are enhanced from time to time. On the other hand, it has a high potential to sink a considerable amount of carbon and hence confront climate change across the globe. For instance, literature review from various previous studies reported that mean carbon storage rate ranges from $30 \mathrm{Mgha}^{-1}$ to $121 \mathrm{Mgha}^{-1}$, while the mean carbon sequestration potential is $6-13 \mathrm{Mgha}^{-1} \mathrm{yr}^{-1}$ [56]. Similarly, the carbon sequestration potential of Moso bamboo is 43 tone $\mathrm{ha}^{-1}$ [8]. This, in turn, plays a paramount importance in the Clean Development Mechanism (CDM) and Climate Resilient Green Economy (CRGE).

6.2. Challenges and Constraints. Despite immense opportunities of the resource, there are critical challenges faced to the bamboo resource in the African region. Our extensive literature review reported that data are almost unavailable, fragmented, inconsistent, and even contradictory [7]. For instance, the study further noted that out of 7 introduced bamboo species in Algeria, only 1 species is identified and included during this review. In addition, the resource is marginalized and neglected by development practitioners so that its utilization is restricted to traditional and cultural uses in the rural setting. Its importance and use are limited to hut construction, fencing, production of handicrafts (basketry, mats, hats, and decorative items), water pipes, furniture, and other household utensils. Among others, common occurrence in the river banks, stream banks, pocket areas, hillsides, between fields and abandoned areas, degraded areas, and planted as hedges are some of the existing evidences. Due to this reason, there are limited management practices, and hence, depletion of bamboo resource is the major concern in potential areas. Likewise, the resource is gradually declining due to various human-induced and natural factors. These include agricultural expansion or shifting cultivation, high fuel wood demand, construction and human settlement, and other associated factors $[7,13,14,36,39,42]$. Uncontrolled and/or deliberate forest fire in the dryland areas, overgrazing/over browsing by livestock particularly during dry seasons in lowland areas (O. abysinica) or in limited feed resource in the highland areas (O. alpina), and overharvesting the resource further aggravate the problem. Furthermore, limited availability of seeds; difficulty in seed collection, processing, and handling; low seed viability; and poor seed storage characteristics are the practical problems in bamboo large-scale propagation using seeds $[40,47]$. The problem is even more complicated with mass flowering and death of bamboo (Figures 5(a) and 5)(b), flowering at longer intervals, and unpredicted flowering $[29,38,39,42]$. Overall, all the aforementioned limitations influence the small-scale and large-scale plantation expansion and development, sustainable use and management, as well as genetic resource conservation of the species. Therefore, it needs urgent call for special focus and action for the sustainable development and promotion of bamboo resource in the African region.

\section{Successful Achievements on Bamboo Resource in the African Region}

Despite the long history of bamboo resource in the African region, bamboo processing and utilization are at the infant stage. However, bamboo processing and utilization in Ethiopia have some base and more competitive than other African countries [57]. The same author reported that there are above 100 bamboo furniture enterprises in Ethiopia with high quality and well-designed products. This author also noted that four modem enterprises produce bamboo floor, door, curtain, charcoal, and other products in Ethiopia. Currently, some successful achievements have been conducted on bamboo development and promotion in the region. First, an international intergovernmental organization, i.e., International Network for Bamboo and Rattan (INBAR) was established in 1997 between China and Africa for the sustainable development of bamboo and rattan in Africa [12]. With this opportunity, 40 African countries are involved in this international cooperation and exchange between China and bamboo-growing countries [27]. Following 
this cooperation, several African nations have participated on short-term and long-term training and awareness raising opportunity on bamboo propagation, cultivation, and bamboo management. In relation to this, developing national bamboo policy in Kenya [58], national bamboo strategy and action plan in Ethiopia [51] and Uganda [59], as well as bamboo policy integration analysis in Ghana [60] are typical actions of bamboo development, promotion, and commercialization in the African region. In the same way, resource base inventory, introduction of new species from bamboo potential regions, propagation, cultivation, management, and sustainable utilization of bamboo resource in the African region become more strengthened. In relation with better awareness raising on bamboo development and promotion, some bamboo processing enterprises, cooperatives, and private industries and factories are emerged, providing various bamboo end products to either local or international markets. For instance, Bamboo Star AgroForestry Company and other bamboo factories and enterprises in Ethiopia are recently emerged and established for processing and producing bamboo endproducts either for local or international markets. Some of these products are production of toothpicks, chopsticks, and household furniture (table, door, and chair). Bamboo culms for scaffolding, casting concrete flooring, building and construction industry, pulp and paper production, laminated boards, and timber production by replacing forest wood in Africa are still new emerging experiences and skills. Furthermore, considering its immense socioeconomic, cultural, and ecological significances, various mega research projects have been initiated and implemented by some African countries. Among these, research projects on bamboo propagation, cultivation, management, and sustainable utilization as well as mass flowering and death of indigenous bamboo species in Ethiopia have been initiated and implemented formerly by the Ethiopian Institute of Agricultural Research (EIAR) and recently succeeded by Ethiopian Environment and Forest Research Institute (EEFRI) as typical model examples. In line with this, 25 bamboo species are introduced from different countries [15], and species adaptation trail has been conducted at different agroecologies [61, 62]. These species are Dendrocalamus asper, D. hamiltonii, D. membranaceus, Bambusa vulgaris var. green, B. vulgaris var. vittata, and Bambusa balcooa [61]. Of these, D. hamiltonii, D. membranaceus $[61,62]$, D. asper, and Bambusa vulgaris var. green [37] are the best adapted species at field. Similarly, related bamboo research studies are carried out by different researchers and professionals on indigenous and/or introduced bamboo species. Some of these are bamboo resource base assessment [35], seed propagation [40, 44-47], seedling performance [44-46, 63], utilization and management $[37,61,64-66]$, vegetative propagation $[41,48,67,68]$, nutritional contents of shoots [55], and their physicochemical features $[69,70]$ of O. alpina and/or O. abyssinica edible shoots. Other research outputs include suitability of bamboo species for construction [50, 71-73], paper and panel boards [73, 74], furniture [39, 42, 70-74], and handcrafts $[39,42,73,74]$, industries, chemical and biochemical industries [75], as well as bioenergy production (charcoal and briquette) and durability of bamboo culms against biodegradable agents and its control measures $[76,77]$. Moreover, comprehensive socioeconomic assess mentions indigenous bamboo species [52, 54, 78-91], and multiplication, prescaling up, and promotion of successfully adapted introduced bamboo species (e.g., Dendrocalamus hamiltonii) [61] are further achievements of bamboo research in Ethiopia. On the other hand, similar or related bamboo research studies have been conducted in different African countries at different times by different professionals. Some of these are Benin [92], Cameroon [23], Ghana [26, 93-97], Kenya [21, 24, 28, 29, 35], Nigeria [98-102], Malawi [31], Tanzania [103], Togo [33], and Uganda $[35,104,105]$.

\section{Conclusion and Recommendations}

Our extensive literature review clearly showed that the African region has untapped bamboo resource potential with immense socioeconomic, cultural, and ecological significances. However, this resource is depleted as a faster rate associated with human-induced and natural factors. In addition, there are no reliable and accurate resource base data due to the lack of well-defined definition and comprehensive resource base inventory in the region. Hence, the information is inaccessible, fragmented, inconsistent, and even contradictory. Therefore, comprehensive research and accurate baseline information on bamboo resource is still required as a foundation for policy and management decisions. Similarly, most of the bamboo resource in the region is either public or state property, so that special focus and appropriate management intervention are not practiced. Hence, the ownership right on bamboo resource and associated land is also clearly specified and certified. At the same time, various silvicultural applications such as propagation, stand density management, fertilizer application, research on mass flowering and death of bamboo and its longer flowering cycle, and preharvesting and postharvesting technologies should be implemented for higher bamboo end products (timber, bioenergy, and edible shoot). Similarly, genetic resource conservation of bamboo species through ex situ conservation (e.g., seed storage in cold room at $+5 \mathrm{C}$ ) and in situ conservation (establishing bamboo botanic garden at field) is also practiced despite the little effort. In line with this, establishing bamboo research institutions and stakeholders, community-based bamboo cooperatives and enterprisers, bamboo industries and factories, as well as small-scale and large-scale bamboo investors/farmers in plantation development should be further strengthened on bamboo resource development and promotion in the region.

\section{Conflicts of Interest}

The authors declare that there are no conflicts of interest. 


\section{References}

[1] D. Ohrnberger, The Bamboos of the World: Annotated Nomenclature and Literature of the Species and the Higher and Lower Taxa, Elsevier, Amsterdam, Netherlands, 1999.

[2] S. Phillips, "Poaceae (Gramineae)," in Flora of Ethiopia Volume 7, I. Hedberg and S. Edwards, Eds., pp. 3-6, Uppsala University, Uppsala, Sweden, 1995.

[3] Z. Wu, P. H. Raven, and D. Hong, Flora of China, Vol. 22 (Poaceae), Science Press, Beijing and Missouri Botanical Garden Press, St. Louis, MO, USA, 2006.

[4] T. J. Meredith, Bamboo for Gardens, Timber Press, Portland, OR, USA, 2001.

[5] M. Fu, Sustainable Management and Utilization of Sympodial Bamboos, China Forestry Publishing House, Beijing, China, 2007.

[6] Z. Peng and X. Wan, "Bamboo culture," in Bamboo and Rattan in the World, Z. Jiang, Ed., pp. 246-256, China Forestry Publishing House, Beijing, China, 2007.

[7] FAO, World Bamboo Resources: A Thematic Study Prepared in the Framework of the Global Forest Resources Assessment 2005, Non-Wood Forest Products No. 18, Food and Agriculture Organization of the United Nations, Rome, Italy, 2007.

[8] Y. Lou, "Comprehensive benefits of Bamboo forests," in Bamboo and Rattan in the World, Z. Jiang, Ed., pp. 106-114, China Forestry Publishing House, Beijing, China, 2007.

[9] UNDPI, "Bamboo: Africa's untapped potential," AfricaRenewal, vol. 30, no. 1, , 2016, http://www.un.org/africarenewal.

[10] X. Chen, J. Dong, H. Dai et al., Technical Manual on Asian Tropical Bamboo Shoots Production, Processing and Marketing, China Forestry Publishing House, Beijing, China, 2010.

[11] M. Fu, J. Xie, B. Zhou, Z. Li, and X. Xiao, Technical Manual on Symopodial Bamboos Cultivation, China Forestry Publishing House, Beijing, China, 2007.

[12] Z. Jiang and X. Liu, "Introduction," in Bamboo and Rattan in the World, Z. Jiang, Ed., pp. 2-9, China Forestry Publishing House, Beijing, China, 2007.

[13] K. Embaye, "The indigenous bamboo forests of Ethiopia: an overview," AMBIO: A Journal of the Human Environment, vol. 29, no. 8, pp. 518-521, 2000.

[14] K. Embaye, "The Indigenous Bamboos of Ethiopia: a call for attention and action," Walia:journal of the Ethiopian Wildlife and Natural History Society, vol. 20, pp. 3-8, 1999.

[15] H. Huojin, Monograph on Bamboo Resources and Utilization Techniques, Ministry of Environment and Forest, Mishqen Printing Press, Addis Ababa, Ethiopia, 2014a.

[16] F. Maina, Selected Works of Bamboo Research and the Picture of Bamboo, The Bamboo Research Editorial Committee. Nanjing Forestry University, Nanjing, China, 2005.

[17] INBAR, "World checklist of bamboos and rattans," INBAR Technical Report No. 37, INBAR, Beijing, China, 2016.

[18] N. Bystriakova, V. Kapos, and I. Lysenko, BambooBiodiversity, UNEP-WCMC/INBAR, Cambridge, UK, 2004.

[19] W. D. Clayton, M. S. Vorontsova, K. T. Harman, and H. Williamson, Grass Base-The Online World Grass Flora, http://www.kew.org/data/grasses-db.html, January 2020.

[20] T. Inada and J. B. Hall, "Oxytenanthera abyssinica (A. Rich.) Munro," in PROTA (Plant Resources of Tropical Africal Ressources végétales de l'Afrique tropicale), Wageningen, D. Louppe, A. A. Oteng-Amoako, and M. Brink, Eds., , http://www.prota4u.org/search.asp, March 2018.
[21] J. M. Grimshaw, "The afromontane bamboo, Yushania alpina, on Kilimanjaro," Journal of East African Natural History, vol. 88, no. 1, pp. 79-83, 1999.

[22] INBAR, Bamboo as Sustainable Biomass Energy. Newsletter No. 2, INBAR, Beijing, China, 2010a.

[23] V. Ingram, J. C. Tieguhong, E. M. Nkamgnia, J. P. Eyebe, and N. Ngawel, Bamboo Production to Consumption System in Cameroon, (Working Paper No. 50), CIFOR/INBAR, Yaounde, Cameroon, 2010.

[24] KFRI, Status of Bamboo Resources Development in Kenya, Kenya Forestry Research Institute (KFRI), Nairobi, Kenya, 2008.

[25] S. F. Moustafa, O. G. Mohamed, M. M. Fathy et al., "Botanical and genetic characteristics of certain Bambusa species cultivated in Egypt," World Journal of Pharmacy and Pharmaceutical Sciences, vol. 3, no. 6, pp. 2075-2100, 2014.

[26] E. Appiah-Kubi, F. W. Owusu, S. L. Tekpetey, C. Essien, and H. Seidu, "Investigating the mechanical properties of some bamboo species for efficient utilization in Ghana," Journal of Bamboo and Rattan, vol. 13, pp. 81-89, 2014.

[27] INBAR, "Bamboo for Africa: a strategic resource to drive the continent's green economy," Policy Synthesis Report No. 2, INBAR, Beijing, China, 2015.

[28] B. N. Kigomo and J. F. Kamiri, "Observations on the growth and yield of Oxytenanthera abyssinica (A. Rich) Munro in plantation," East African Agricultural and Forestry Journal, vol. 51, no. 1, pp. 22-29, 1985.

[29] B. N. Kigomo, Guidelines for Growing Bamboo. KEFRI Guideline Series: No. 4, KenyaForestry Research Institute (KEFRI). Downtown Printing Works Ltd., Nairobi, Kenya, 2007.

[30] T. King, H. L. L. Randrianarimanana, L. H. F. Rakotonirina et al., "Large-culmed bamboos in Madagascar: distribution and field identification of the primary food sources of the critically endangered greater bamboo LemurProlemur simus," Primate Conservation, vol. 27, no. 1, pp. 33-53, 2013.

[31] B. G. Sosola-Banda and F. H. Johnsen, "Rural livelihoods on Bamboo handicraft making and culm vending in Mvera, Malawi," Journal of Bamboo and Rattan, vol. 4, no. 1, pp. 93-107, 2005.

[32] R. Haroun, A. H. Barrencua, and A. D. Abreu, "Mangrove habitats in São Tomé and Príncipe (Gulf of Guinea, Africa): conservation and management status," in Threats to Mangrove Forests, Coastal Research Library 25, C. Makowski and C. W. Finkl, Eds., Springer International Publishing AG, Part of Springer Nature, Basingstoke, UK, pp. 589-605, 2018.

[33] A. Randriahaingo, K. Adjonou, A. Guelly, and K. Kokou, "Bamboo resources in Togo," International Journal of Biological and Chemical Sciences, vol. 8, no. 2, pp. 481-493, 2014.

[34] INBAR, "Properties of East African bamboo: the physical, mechanical, chemical and morphological test results of three East African bamboo species," INBAR Working Paper No. 80, Beijing, China, 2019.

[35] Y. Zhao, D. Feng, D. Jayaraman et al., "Bamboo mapping of Ethiopia, Kenya and Uganda for the year 2016 using multi-temporal Landsat imagery," International Journal of Applied Earth Observation and Geoinformation, vol. 66, pp. 116-125, 2018.

[36] FAO, "Global forest resources assessment 2010 main report," Food and Agriculture Organization of the United Nations, Rome, Italy, FAO Forestry Paper No. 163, 2010.

[37] Y. Mulatu, A. Alemayehu, and Z. Tadesse, Biology and Management of Indigenous Bamboo Species of Ethiopia: Based on Research and Practical Field Experience, Ethiopian 
Environment and Forest Research Institute (EEFRI), Addis Ababa, Ethiopia, 2016b.

[38] D. Sertse, T. Disasa, K. Bekele et al., "Mass flowering and death of Bamboo: a potential threat to biodiversity and livelihoods in Ethiopia," Journal of Biodiversity and Environmental Sciences, vol. 1, no. 5, pp. 16-25, 2011.

[39] UNIDO, Bamboo Cultivation Manual. Guidelines for Cultivating Ethiopian Highland Bamboo, Eastern Africa Bamboo Project, United Nations Industrial Development Organization (UNIDO), Vienna, Austria, 2009a.

[40] T. Bahru, Y. Mulatu, and B. Kidane, "Germination ecology of Arundinaria alpina (K. Schum.) and Oxytenanthera abyssinica (A. Rich.) Munro seeds: indigenous bamboo species in Ethiopia," International Journal of Biodiversity, vol. 2015, Article ID 323128, 8 pages, 2015.

[41] T. Hunde, Y. Adane, and M. Adilo, "Assessment of different vegetative propagation techniques on rooting and shooting ability of Arundinaria alpina," Ethiopian Journal of Natural Resources, vol. 7, no. 1, pp. 131-139, 2005.

[42] UNIDO, Bamboo Cultivation Manual. Guidelines for Cultivating Ethiopian Lowland Bamboo, Eastern Africa BambooProject, United Nations Industrial Development Organization (UNIDO), Vienna, Austria, 2009b.

[43] S. Nune, "Short notes on bamboo," in Kosso, A Quarterly Newsletter of the Ethiopian Foresters' Association, pp. 2-5, Ethiopian Foresters' Association (EFA), Addis Ababa, Ethiopia, 2001.

[44] D. A. Ayana, "Seed Characterstics of Lowland Bamboo (Oxytenanthera abysinica (A. Rich.) Munro)) as affected by site and position of fruit on the culm," M.Sc. thesis, Hawassa University, Shashemene, Ethiopia, 2006.

[45] D. A. Ayana, A. Gure, and K. Embaye, "Flowering and causes of seed defects in lowland bamboo (Oxytenanthera abyssinica): a case study in Benishangul Gumuz Regional State, Northwestern Ethiopia," International Journal Life Sciences, vol. 4, no. 4, pp. 251-259, 2015.

[46] T. Bahru, Y. Mulatu, and B. Kidane, "Provenance variation on early survival rate and growth performance of Oxytenanthera abyssinica (A. Rich.) Munro seedlings at greenhouse: an indigenous lowland bamboo species in Ethiopia," International Journal of Energy Research, vol. 2018, Article ID 5713456, 2018.

[47] T. Bahru, B. Kidane, A. Araya et al., "Effects of germination sites on germination percentage, germination energy and germination value of the lowland Bambooseeds," in Forestry and Forest Products in Ethiopia. Proceedings of the National Workshop on Forestry Research Technologies Dissemination 29-31 May 2012, Hiruy Hall, W. Tadesse, G. Desalign, and A. Yirgu, Eds., pp. 85-95, Ethiopian Institute of Agricultural Research (EIAR), Addis Ababa, Ethiopia, 2012.

[48] B. Kahsay, F. Mekibib, and A. Teklewold, "In vitro propagation of Oxytenanthera abyssinica (A. Rich. Munro) from seed culture," Biotechnology Journal International, vol. 18, no. 2, pp. 1-13, 2017.

[49] R. Sharma, J. Wahono, and H. Baral, "Bamboo as an alternative bioenergy crop and powerful ally for land restoration in Indonesia," Sustainability, vol. 10, no. 12, p. 4367, 2018.

[50] M. Abegaz, N. Jiru, and B. Oluma, "Oxytenanthera abyssinicabamboo stems as reinforcement steel bar in concrete," Ethiopian Journal of Natural Resources, vol. 7, no. 1, pp. 95-109, 2005.

[51] EBDSAP, 2019-2030 Ethiopian Bamboo Development Strategy and Action Plan,Environment, Forest and Climate
Change Commission of Ethiopia \& International Bamboo and Rattan Organisation (INBAR), Addis Ababa, Ethiopia, Beijing, China, 2020.

[52] T. Teshale, T. Woldeamanuel, T. Bekele, A. Alemu, and J. Pretzsch, "Market channels for highland bamboo poles originated from Hula district, Sidama zone southern Ethiopia," Small-Scale Forestey, vol. 16, no. 3, 2017.

[53] T. Endalamaw, A. Lindner, and J. Pretzsch, "Indicators and determinants of small-scale bamboo commercialization in Ethiopia," Forests, vol. 4, no. 3, pp. 710-729, 2013.

[54] Z. Mekonnen, A. Worku, T. Yohannes, M. Alebachew, D. Teketay, and H. Kassa, "Bamboo resources in Ethiopia: their value chain and contribution to livelihoods," Ethnobotany Research and Applications, vol. 12, pp. 511-524, 2014.

[55] Y. Mulatu, T. Bahru, B. Kidane, A. Getahun, and A. Belay, "Proximate and mineral composition of indigenous bamboo shoots of Ethiopia," Greener Journal of Agricultural Sciences, vol. 9, no. 2, pp. 215-221, 2019.

[56] A. J. Nath, R. Lal, and A. K. Das, "Managing woody bamboos for carbon farming and carbon trading," Global Ecology and Conservation, vol. 3, pp. 654-663, 2015.

[57] H. Huojin, "Some proposals for developing Ethiopian bamboo industry," in Suggestions for the Development of Agriculture and Forestry in Ethiopia, pp. 50-54, China Agriculture Expertise Team in Ethiopia, Addis Ababa, Ethiopia, 2014 b.

[58] NBP, National Bamboo Policy, 2019, Ministry of Environment and Forestry, Nairobi, Kenya, 2019.

[59] UNBSAP, 2019-2029 Uganda National Bamboo Strategy and Action Plan, Ministry of Water and Environment of Uganda \&International Bamboo and Rattan Organisation (INBAR), Kampala, Uganda \&, Beijing, China, 2020.

[60] INBAR, "Bamboo Policy Integration Analysis in Ghana," INBAR, Beijing, China, INBAR Working Paper No. 83, 2020.

[61] Y. Mulatu, A. Alemayehu, and Z. Tadesse, Bamboo Species Introduced in Ethiopia: Biological, Ecological and Management Aspects, Ethiopian Environment and Forest Research Institute (EEFRI), Addis Ababa, Ethiopia, 2016a.

[62] R. Terefe, D. Samuel, M. Sanbato, and M. Daba, "Adaptation and growth performance of different lowland bamboo species in Bako, West Shoa, Ethiopia," Journal of Natural Sciences Research, vol. 6, no. 9, pp. 61-65, 2016.

[63] K. Embaye, L. Christersson, S. Ledin, and M. Weih, "Bamboo as bioresource in Ethiopia: management strategy to improve seedling performance (Oxytenanthera abyssinica)," Bioresource Technology, vol. 88, no. 1, pp. 33-39, 2003.

[64] S. Gebrekidan, L. Tiki, and Y. Mulatu, "Indigenous knowledge on highland bamboo (Yushania alpina) management and utilization practices in Kokosa Woreda, South East Ethiopia," Scientific Research and Essays, vol. 13, no. 11, pp. 111-122, 2018.

[65] INBAR, Study on Utilization of Lowland Bamboo in Benishangul Gumuz Region, Ethiopia, INBAR, Beijing, China, 2010b.

[66] U. Teshoma, "Carbon storage potential of Ethiopian highland bamboo (Arundinaria alpina (K. schum): a case study of Adiyo Woreda, South West Ethiopia," International Journal of Environmental Sciences \& Natural Resources, vol. 16, no. 5, pp. 001-011, 2019.

[67] Y. Kebede, Z. Tadesse, A. Getahun, and Y. Mulatu, "Vegetative propagation techniques of highland bamboo (Yushania alpina) in Amhara Region, North-Western Ethiopia," World Scientific News, vol. 61, no. 2, pp. 122-136, 2017. 
[68] Y. Mulatu and A. Alemayehu, Propagation of Bamboo Species in Ethiopia: Based on Practical Field Experience and Research, Ethiopian Environment and Forest Research Institute (EEFRI), Addis Ababa, Ethiopia, 2016.

[69] S. Feleke, K. Tesfaye, and A. Tebeje, "Physicochemical characteristics of bamboo shoots from Yushania alpina and Oxytenanthera abyssinica growing in Ethiopia," in Forestry and Forest Products in Ethiopia. Proceedings of the National Workshop on Forestry Research Technologies Dissemination 29-31 May 2012, W. Tadesse, G. Desalign, and A. Yirgu, Eds., pp. 294-300, Hiruy Hall, Ethiopian Institute of Agricultural Research (EIAR), Addis Ababa, Ethiopia, 2012.

[70] O. Obsa, M. Kassa, and L. Tajebu, "Physicochemical properties of bamboo (Arundinaria alpina) based agroforestry practice in Dawuro Zone, South West Ethiopia," Journal of Biology, Agriculture and Healthcare, vol. 5, no. 9, pp. 104-109, 2015b.

[71] S. Kelemwork, "Influence of growing locations and culm positions on physical and mechanical properties of lowland bamboo (Oxythenantera abyssinica)," in Forestry and Forest Products in Ethiopia. Proceedings of the National Workshop on Forestry Research Technologies Dissemination 29-31 May 2012, Hiruy Hall, W. Tadesse, G. Desalign, and A. Yirgu, Eds., pp. 317-327, Ethiopian Institute of Agricultural Research (EIAR), Addis Ababa, Ethiopia, 2012.

[72] S. Kelemwork, "Effects of anatomical characteristics of Ethiopian lowland bamboo on physical and mechanical properties," China Association for Science and Technology, vol. 8, no. 3, pp. 32-43, 2009.

[73] S. Kelemwork, P. M. Tahir, W. E. Ding, and R. Sudin, "The effects of selected anatomical characterstics on physical properties of Ethiopian highland bamboo Arundinaria alpina K.Schum. (Poaceae)," Ethiopian Journal of Biological Sciences, vol. 7, no. 1, pp. 69-84, 2008.

[74] Y. Gebremariam and D. Assefa, "Effect of age and height on some selected physical properties of Ethiopian highland bamboo (Yushania alpina)," International Journal of Scientific Research and Management, vol. 6, no. 8, pp. 70-74, 2018.

[75] A. Tolessa, B. Woldeyes, and S. Feleke, "Chemical composition of lowland bamboo (Oxytenanthera abyssinica) grown around Asossa Town, Ethiopia," World Sci. News, vol. 74, pp. 141-151, 2017.

[76] G. Desalegn, "Durability of Ethiopian bamboo culms and alternative damage control measures against biodeteriorating agents," Ethiopian Journal of Science and Technology, vol. 14, no. 2, pp. 93-127, 2015.

[77] G. Desalegn and M. Abegaz, "Increasing the service life of bamboo culms as construction and furniture material by controlling biodeterioration and rational utilization," in Forestry and Forest Products in Ethiopia. Proceedings of the National Workshop on Forestry Research Technologies Dissemination 29-31 May 2012, Hiruy Hall, W. Tadesse, G. Desalign, and A. Yirgu, Eds., pp. 328-360, Ethiopian Institute of Agricultural Research (EIAR), Addis Ababa, Ethiopia, 2012.

[78] A. Andargatchew, "Value chain analysis for bamboo originating from Shedem Kebele, Bale Zone," M.Sc. thesis, Addis Ababa University, Addis Ababa, Ethiopia, 2000.

[79] F. Bakala, T. Bekele, and T. Woldeamanuel, "Market supply determinants of lowland bamboo culms: the case of Homosha district, Northwestern Ethiopia," African Journal of Marketing Management, vol. 9, no. 4, pp. 46-58, 2017.

[80] S. Erifo, L. Zemedu, and T. W/amanuel, "Value chain analysis of bamboo production: the case of Bule Woreda,
Gedeo Zone," Industrial Engineering Letters, vol. 6, no. 1, pp. 38-47, 2016.

[81] M. Fekadu, E. Csaplovics, and P. Degeen, "Household contribution of bamboo in Masha district, southern Ethiopia," in Forestry and Forest Products in Ethiopia. Proceedings of the National Workshop on Forestry Research Technologies Dissemination 29-31 May 2012, Hiruy Hall, W. Tadesse, G. Desalign, and A. Yirgu, Eds., pp. 263-273, Ethiopian Institute of Agricultural Research (EIAR), Addis Ababa, Ethiopia, 2012.

[82] G. D. Gebramlak, N. Abadi, and E. B. Hizikias, "Socioeconomic contribution of Oxytenanthera abyssinica (A. Rich) Munro and determinants of growing in homestead agroforestry system in northern Ethiopia," Ethnobotany Research and Applications, vol. 14, pp. 479-490, 2016.

[83] Y. W. Guadie, D. H. Feyssa, and D. B. Jiru, "Socio-economic importance of highland bamboo (Yushania alpina K. Schum) and challenges for its expansion in Bibugn District, East Gojjam, Ethiopia," Journal of Horticulture and Forestry, vol. 11, no. 2, pp. 32-41, 2019.

[84] T. Kassahun, "Review of bamboo value chain in Ethiopia," Journal of Biology, Agriculture and Healthcare, vol. 4, no. 27, pp. 179-190, 2014.

[85] T. Kassahun, B. Emana, and A. Mitiku, "Determinants of highland bamboo (Yushania alpina) culm supply: the case of Loma and Tocha districts, Dawuro Zone of Southern Ethiopia," Journal of Biology, Agriculture and Healthcare, vol. 5, no. 21, pp. 49-60, 2015.

[86] A. A. Kebede, "Opportunities and challenges to highland bamboo-based traditional handicraft production, marketing and utilization in Awi Zone, Northwestern Ethiopia," International Journal of History and Cultural Studies, vol. 4, no. 4, pp. 57-67, 2018.

[87] E. Kelbessa, T. Bekele, A. Gebrehiwot, and G. Hadera, A Socio-Economic Case Study of the Bamboo Sector in Ethiopia: An Analysis of the Production-to-Consumption System, International Bamboo and Rattan Organisation, Addis Ababa, Ethiopia, 2000.

[88] Y. Mekuriaw, M. Urge, and G. Animut, "Role of indigenous bamboo species (Yushania alpina and Oxytenanthera abyssinica) as ruminant feed in northwestern Ethiopia," Livestock Research for Rural Development, vol. 23, no. 9, p. 2011, 2011.

[89] O. Obsa, M. Kassa, and L. Tajebu, "Income contribution of bamboo (Arundinaria alpina) based agroforestry practice in Dawuro Zone, South West Ethiopia," Journal of Economics and Sustainable Development, vol. 6, no. 9, pp. 155-161, $2015 \mathrm{a}$.

[90] K. Oyicha, Afromontane Bamboo Arundinaria alpina in Sidama Highlands: Its Utilization and Propagation Capacity by Culm Cuttings, MSc. thesis, Swedish University of Agricultural Sciences, Skinnskatteberg, Sweden, 1997.

[91] B. L. Tinsley, "Bamboo harvesting for household income generation in the ethiopian highlands: current conditions and management challenges," M.Sc. thesis, The University of Montana, Missoula, MT, USA, 2014.

[92] H. Honfo, C. Gnanglè, S. Mensah et al., "Traditional ecological knowledge and use value of bamboo in Southeastern Benin: implications for sustainable management," Ethnobotany Research and Applications, vol. 14, pp. 139-153, 2015.

[93] S. D. Akoto, R. Obour, M. A. Appiah, and A. P. Frimpong, "Bamboo use for the housing industry in Ghana: urban stakeholders' perception," JENRM, vol. 3, no. 3, pp. 85-91, 2017. 
[94] B. D. Obiri and A. A. Oteng-Amoako, "Towards a sustainable development of the bamboo industry in Ghana," Ghana Journal of Science, vol. 21-22, pp. 14-27, 2007.

[95] F. W. Owusu, E. Appiah-Kubi, S. L. Tekpetey, C. Essien, P. I. Arthur, and G. K. Zorve, "Products development of laminated panel doors from plantation grown bamboo species in Ghana," Journal of Bamboo and Rattan, vol. 13, no. 3-4, pp. 91-105, 2014.

[96] S. Rudolf and T. Emmanuel, "Bamboo, hope for the wood industry in Ghana," Journal of Natural Sciences Research, vol. 3, no. 12, pp. 1-5, 2013.

[97] D. Safro, Bamboo as a Source of Bioenergy Feedstock in Ghana, M.Sc. thesis, Kwame Nkrumah University of Science and Technology, Kumasi, Ghana, 2008.

[98] O. Abimbola, "Promoting green growth of forest products industry in Nigeria through bamboo development," Developing Country Studies, vol. 2, no. 11, pp. 65-74, 2012.

[99] J. Atanda, "Environmental impacts of bamboo as a substitute constructional material in Nigeria," Case Studies in Construction Materials, vol. 3, pp. 33-39, 2015.

[100] O. R. Ogbanje and C. M. Diko, "Nigeria and bamboo plants as a forest product," Journal of Agriculture and Veterinary Science, vol. 3, no. 6, pp. 55-59, 2013.

[101] A. A. Ogunjinmi, H. M. Ijeomah, and A. A. Aiyeloja, "Socioeconomic importance of bamboo (Bambusa vulgaris) in Borgu local government area of Niger State, Nigeria," Journal of Sustainable Development in Africa, vol. 10, no. 4, pp. 284-298, 2009.

[102] A. A. Ogunwusi and A. P. Onwualu, "Prospects for multifunctional utilization of bamboo in Nigeria," Chemistry and Materials Research, vol. 3, no. 8, pp. 58-70, 2013.

[103] INBAR, "Rural enterprise development for livelihood enhancement: creation of rural community micro and small enterprises in Tanzania. INBAR's Livelihood Development Programme," 2008, http://www.inbar.int/livelihood/ldmain. htm.

[104] R. Glèlè Kakaï and A. MacNeilage, "Population structure of montane bamboo and causes of its decline in echuya central forest reserve, south West Uganda," African Journal of Ecology, vol. 46, pp. 325-332, 2007.

[105] J. Wong, "Recommendations for the inclusion of nationally important NTFPs in standard forest inventories (EI \& ISSMI)," Report to the EDF/Uganda Forest Department: Forest Resources Management and Conservation Programme, Wild Resources Limited, Gwynedd, UK, 2003. 